\title{
Genome-wide identification of ABA receptor PYL family and expression analysis of PYLs in response to ABA and osmotic stress in Gossypium
}

\author{
Gaofeng Zhang ${ }^{1}$, Tingting Lu $^{1,2}$ ， Wenwen Miao ${ }^{1}$ ， Lirong Sun ${ }^{1}$, Mi Tian ${ }^{1}$ ， Ji Wang ${ }^{1}$, Fushun Hao ${ }^{\text {Corresp. } 1}$ \\ ${ }^{1}$ State Key Laboratory of Cotton Biology, Henan Key Laboratory of Plant Stress Biology, College of Life Sciences, Henan University, Kaifeng, Henan, China \\ 2 Henan University of Animal Husbandry and Economy, Zhengzhou, Henan, China \\ Corresponding Author: Fushun Hao \\ Email address: haofsh@126.com
}

Abscisic acid (ABA) receptor pyrabactin resistance1/PYR1-like/regulatory components of ABA receptor (PYR1/PYL/RCAR) (named PYLs for simplicity) are core regulators of ABA signaling, and have been well studied in Arabidopsis and rice. However, knowledge is limited about PYL family regarding genome organization, gene structure, phylogenesis, gene expression and protein interaction with downstream targets in Gossypium. A comprehensive analysis of Gossypium PYL family was carried out, and 21, 20, 40 and 39 PYL genes were identified in the genomes from the diploid progenitor $G$. arboretum, $G$. raimondii and the tetraploid G. hirsutum and G. barbadense, respectively. Characterization of the physical properties, chromosomal locations, structures and phylogeny of these family members revealed that Gossypium PYLs were quite conservative among the surveyed cotton species. Segmental duplication might be the main force promoting the expansion of $P Y L s$, and majority of the $P Y L s$ underwent evolution under purifying selection in Gossypium. Additionally, the expression profiles of GhPYL genes were specific in tissues. Transcriptions of many GhPYL genes were inhibited by ABA treatments and induced by osmotic stress. A number of GhPYLs can interact with GhABIIA or GhABID in the presence and/or absence of ABA by the yeast-two hybrid method in cotton. 
1 Title page

2 Title:

3 Genome-wide identification of ABA receptor PYL family and expression analysis of $P Y L s$ in

4 response to $\mathrm{ABA}$ and osmotic stress in Gossypium

5 Authors:

6 Gaofeng Zhang ${ }^{1^{*}}$, Tingting $\mathrm{Lu}^{1,2^{*}}$, Wenwen Miao ${ }^{1}$, Lirong Sun ${ }^{1}$, Mi Tian ${ }^{1}$, Ji Wang ${ }^{1}$ and Fu-

7 Shun Hao ${ }^{1}$

$8 \quad{ }^{1}$ State Key Laboratory of Cotton Biology, Henan Key Laboratory of Plant Stress Biology,

9 College of Life Sciences, Henan University, Kaifeng, China

$10 \quad{ }^{2}$ Henan University of Animal Husbandry and Economy, Zhengzhou, China

$11{ }^{*}$ These authors contributed equally to this work

12 Corresponding author

13 Fu-Shun Hao, haofsh@henu.edu.cn 
14 Genome-wide identification of ABA receptor PYL family and expression analysis of PYLs in response to $\mathrm{ABA}$ and osmotic stress in Gossypium

Authors:

Gaofeng Zhang ${ }^{1 *}$, Tingting Lu ${ }^{1,2^{*}}$, Wenwen Miao ${ }^{1}$, Lirong Sun ${ }^{1}$, Mi Tian ${ }^{1}$, Ji Wang ${ }^{1}$ and FuShun Hao'

${ }^{1}$ State Key Laboratory of Cotton Biology, Henan Key Laboratory of Plant Stress Biology, College of Life Sciences, Henan University, Kaifeng, China

${ }^{2}$ Henan University of Animal Husbandry and Economy, Zhengzhou, China

\section{ABSTRACT}

Abscisic acid (ABA) receptor pyrabactin resistance1/PYR1-like/regulatory components of ABA receptor (PYR1/PYL/RCAR) (named PYLs for simplicity) are core regulators of ABA signaling, and have been well studied in Arabidopsis and rice. However, knowledge is limited about PYL family regarding genome organization, gene structure, phylogenesis, gene expression and protein interaction with downstream targets in Gossypium. A comprehensive analysis of Gossypium PYL family was carried out, and 21, 20, 40 and 39 PYL genes were identified in the genomes from the diploid progenitor G. arboretum, G. raimondii and the tetraploid G. hirsutum and G. barbadense, respectively. Characterization of the physical properties, chromosomal locations, structures and phylogeny of these family members revealed that Gossypium PYLs were quite conservative among the surveyed cotton species. Segmental duplication might be the main force promoting the expansion of PYLs, and majority of the PYLs underwent evolution under purifying selection in Gossypium. Additionally, the expression profiles of GhPYL genes were specific in tissues. 
35 Transcriptions of many GhPYL genes were inhibited by ABA treatments and induced by osmotic

36 stress. A number of GhPYLs can interact with GhABI1A or GhABID in the presence and/or

37 absence of ABA by the yeast-two hybrid method in cotton. 
INTRODUCTION

Abscisic acid (ABA) is one of the most important phytohormones. It regulates multiple cellular processes including seed maturation and dormancy, seedling growth, leaf senescence and stomatal movement in plants (Cutler et al., 2010). Moreover, ABA play crucial roles in plant responses to various stresses such as drought, salinity, osmotic stress, extreme temperature, pathogen attack, and so on (Cutler et al., 2010; Lee et al., 2012). When plants are exposed to stresses, particularly dehydrate stress, the level of ABA in tissues prominently increases. It has been perceived that $\mathrm{ABA}$ can bind to the $\mathrm{ABA}$ receptor pyrabactin resistance1/PYR1like/regulatory components of $\mathrm{ABA}$ receptor (PYR1/PYL/RCAR) proteins (hereafter referred to as PYLs for simplicity). The formed complex interacts with and suppresses the activities of group A type $2 \mathrm{C}$ protein phosphatases ( $\mathrm{PP} 2 \mathrm{Cs}$ ), the key negative regulators of $\mathrm{ABA}$ signaling. Consequently, PP2Cs unbind and activate the sucrose nonfermenting 1-related protein kinases (SnRKs) subfamily 2 (SnRK2s), the pivotal positive regulators of ABA signal cascade. SnRK2s subsequently phosphorylate and modulate downstream transcriptional factors or other proteins to cause physiological responses to ABA (Fujii et al., 2009) (Fig. 1). PYLs, PP2Cs and SnRK2s have been proved to be core components of ABA signaling. PP2Cs mainly include ABI1 (ABA insensitive 1), $\mathrm{ABI}$, $\mathrm{HAB} 1$ (Hypersensitive to $\mathrm{ABA}$ 1), $\mathrm{HAB} 2$, and so forth; and SnRK2s mainly consist of SnRK2.2, SnRK2.3 and SnRK2.6 in Arabidopsis (Ma et al., 2009; Park et al., 2009; Lim et al., 2012).

PYLs belong to the START (Star-related lipid-transfer) superfamily of ligand-binding proteins (Ma et al., 2009; Park et al., 2009). In Arabidopsis, 14 AtPYLs including AtPYR1 (Arabidopsis thaliana pyrabactin resistance1) and 13 AtPYLs (Arabidopsis thaliana PYR1-like1-13) were 
60 found (Ma et al., 2009; Park et al., 2009). Of which, AtPYR1, AtPYL1, AtPYL2 and AtPYL3

61 inhibit PP2Cs in a ABA dependent manner while AtPYL4, AtPYL5, AtPYL6, AtPYL8, AtPYL9,

62 AtPYL10 and AtPYL13 show suppression of PP2Cs in the absence of ABA (Hao et al., 2011; Li

63 et al., 2013; Miyakawa et al., 2013). Genetic evidence indicated that AtPYR1, AtPYL1, AtPYL2,

64 AtPYL4, AtPYL5 and AtPYL8 redundantly play roles in ABA-mediated seed germination, root

65 growth and stomatal closure (Park et al., 2009; Gonzalez-Guzman et al., 2012). AtPYL5 and

66 AtPYL9 regulates drought resistance (Santiago et al., 2009; Zhao et al., 2016). AtPYL9 also

67 modulates leaf senescence (Zhao et al., 2016). Moreover, AtPYL8 and AtPYL9 were reported to

68 function in root development (Antoni et al., 2013; Zhao et al., 2014; Xing et al., 2016). AtPYL6

69 and AtPYL13 were described to positively affect ABA inhibition of seed germination (Fuchs et

70 al., 2014). In addition, it has been addressed that the orthologs of AtPYLs in rice, soybean,

71 tomato, maize, wheat, poplar and Brachypodium distachyon play roles in growth and in response

72 to stresses (Kim et al., 2012; Bai et al., 2013; González-Guzmán et al., 2014; Tian et al., 2015;

73 Fan et al., 2016; Gordon et al., 2016; Yu et al., 2016; Zhang et al., 2017). Very recently, Chen et

74 al. (2017) reported that overexpressing cotton GhPYL10/12/26 in Arabidopsis increased ABA

75 sensitivity during seed germination and seedling growth. Liang et al. (2017) also found that

76 cotton GhPYL9-11A positively regulate drought tolerance in transgenic Arabidopsis plants. In

77 recent years, many PYL gene families have been characterized at genome-wide levels in rice,

78 grape, soybean and other plants (Boneh et al., 2012; Kim et al., 2012; Bai et al., 2013; González-

79 Guzmán et al., 2014; Tian et al., 2015; Fan et al., 2016; Gordon et al., 2016; Yu et al., 2016; Guo

80 et al., 2017; Zhang et al., 2017). Furthermore, the structure properties of AtPYLs in Arabidopsis

81 have been intensively investigated. It is found that an ABA-binding pocket, three $\alpha$-helix, seven $\beta$ 
82 sheets and four loops are conserved in AtPYLs (Ma et al., 2009; Park et al., 2009; Santiago et al.,

83 2012). Similar structures also exist in other plants. Furthermore, the expression patterns of PYL

84 genes have been studied in tissues and in responding to exogenous ABA and diverse stresses in

85 multiple plants (Saavedra et al., 2010; Boneh et al., 2012; Kim et al., 2012; Bai et al., 2013;

86 González-Guzmán et al., 2014; Tian et al., 2015; Fan et al., 2016; Gordon et al., 2016; Yu et al.,

87 2016; Chen et al., 2017; Zhang et al., 2017). These findings provide valuable information for

88 researchers to further examine the functions of PYLs in ABA signaling in plants. However,

89 knowledge about PYLs in Gossypium is scarce.

90 Cotton is the most important fiber crop and a key cash crop in the world. It provides the

spinnable lint for the textile industry. It has been demonstrated that growth and development of

cotton plants are seriously affected by a variety of environmental stresses such as drought, salinity and cold (Allen, 2010). These adverse stresses lead to significant decreases in the yield and quality of cotton fiber worldwide. Accordingly, it is of great importance to enhance stress tolerance of cotton plants. One of the key strategies may be achieved via genetically engineering of PYL genes (Kim et al., 2014; Lee et al., 2015; Yu et al., 2016; Zhao et al., 2016; Chen et al., 2017; Liang et al., 2017). It is therefore essential for us to elucidate the functions and regulatory mechanisms of Gossypium PYLs. Here, we performed genome-wide and comprehensive analyses of the PYL family in $G$. arboretum $\left(\mathrm{A}_{2}\right)$ and $G$. raimondii $\left(\mathrm{D}_{5}\right)$, and their tetraploid species $G$. hirsutum $\left(\mathrm{AD}_{1}\right)$ and $G$. barbadense $\left(\mathrm{AD}_{2}\right)$. The expression patterns of PYL genes were studied in

101 various tissues and in response to $\mathrm{ABA}$ and osmotic stress in G. hirsutum. Moreover, the 102 interactions between individual GhPYLs and GhABI1A or GhABI1D were measured in $G$.

103 hirsutum by the yeast-two hybrid method. These results may pave the way for further 
104 investigating the functions of cotton PYLs in the future.

105 MATERIALS AND METHODS

106 Analysis of the PYL family in cotton

107 The amino acid sequences of 14 AtPYLs were searched in the genome sequence databases of $G$.

108 arboretum (BGI-CGB v2.0 assembly genome), G. raimondii (JGI assembly v2.0 data.), $G$.

109 hirsutum (NAU-NBI v1.1 assembly genome) (www.cottongen.org) and G. barbadense

110 (http://database.chgc.sh.cn/cotton/index.html), respectively. The BLAST program was used with

111 default setting $\left(\right.$ E-value $\left.<\mathrm{e}^{-10}\right)$. The protein sequences of PYLs were pairwisely aligned applying

112 the ClustalW software with default parameters (Larkin et al., 2007). Genes with questionable

113 PYL annotations (i.e. having a typical PYL domain but low E-value or low coverage of a

114 domain) were manually reanalyzed.

115 The properties of PYL proteins were assessed by the online tools ExPaSy

116 (http://web.expasy.org/protparam/). The subcellular localizations of Gossypium PYLs were

117 analyzed on the basis of the information from the website

118 (http://www.csbio.sjtu.edu.cn/bioinf/Cell-PLoc). The locations of cotton PYL genes in

119 chromosomes were determined by the MapInspect software

120 (http://www.mybiosoftware.com/mapinspect-compare-display-linkage-maps.html) and their

121 structures were identified by the GSDS (http://gsds.cbi.pku.edu.cn).

122 The conserved domains in PYLs were confirmed in NCBI

123 (https://www.ncbi.nlm.nih.gov/Structure/cdd/wrpsb.cgi). The motifs in Gossypium PYLs were

124 analyzed by the MEME (meme-suite.org/tools/meme).

125 Phylogenetic analysis of PYLs 
126 The PYL-related databases were downloaded from the websites for Theobroma cacao

127 (http://cocoagendb.cirad.fr), Ricinus communis (http://castorbean.jcvi.org), Vitis vinifera

128 (http://www.genoscope.cns.fr/spip/Vitis-vinifera-e.html), Brachypodium distachyon

129 (http://plants.ensembl.org/Brachypodium_distachyon/Info/Index), Oryza sativa

130 (http://rapdb.dna.affrc.go.jp) and Arabidopsis thaliana (http://www.arabidopsis.org), and four

131 cotton plants described above. Multiple sequence alignments for the protein sequences of PYLs

132 were carried out and phylogenetic trees were constructed following the alignment results using

133 the neighbor joining method (Neighbor-Joining, NJ) and 1,000 bootstrap trials with the Clustal W

134 tool (Larkin et al., 2007) and the MEGA 5.0 software (http://www.megasoftware.net).

\section{Synteny and $\mathrm{Ka} / \mathrm{Ks}$ analysis}

136 The values of nucleotide substitution parameter Ka (non-synonymous) and Ks (synonymous)

137 were counted based on the PAML program (http://abacus.gene.ucl.ac.uk/software/paml.html).

138 The homologous genes were searched by the MCScanx software

139 (http://chibba.pgml.uga.edu/mcscan2), and gene collinearity results were obtained by the

140 CIRCOS program (http://www.circos.ca). The syntenic maps of the PYL genes from $G$.

141 arboretum, $G$. raimondii and $G$. hirsutum were constructed using the circos- $0.69 \pm 3$ software

142 package with default parameters (http://www.circos.ca).

143 Expression analysis of PYL genes in tissues and in response to ABA or osmotic stress

144 To monitor expression levels of PYLs in tissues, samples of roots, stems and leaves were obtained

145 from TM-1 cotton plants grown in soil for $21 \mathrm{~d}$. Flowers were picked in the morning at the first

146 day of anthesis, and fibers at the secondary cell wall stage (about $23 \mathrm{~d}$ post anthesis) were

147 sampled from the ovules. To determine $P Y L$ transcript abundances in responding to ABA or 
148 osmotic stress, cotton plants grew in liquid 1/2 MS medium (Murashige and Skoog, 1962) in a

149 growth chamber (day/night temperature cycle of $28^{\circ} \mathrm{C} / 26^{\circ} \mathrm{C}, 14 \mathrm{~h} \mathrm{light} / 10 \mathrm{~h}$ dark, and about $50 \%$

150 relative humidity) for 3 weeks. Then, the plant leaves were sprayed with $100 \mu \mathrm{M}$ ABA or the

151 roots were treated with 10\% PEG6000 (PEG6000 was added in the liquid medium) for 3, 6, 12

152 and $24 \mathrm{~h}$, respectively. The plants that were not sprayed with ABA or were not treated with

153 PEG6000 were as controls. The roots for treatments and controls were collected, frozen in liquid

154 nitrogen and stored at $-70^{\circ} \mathrm{C}$. About $0.1 \mathrm{~g}$ of samples were ground to a fine powder with a mortar

155 and pestle in liquid nitrogen. Total RNA was extracted from the powder using RNA Pure Plant

156 Kit's protocol (TIANGEN Company). The cDNA was obtained by M-MLV reserve transcriptase

157 synthesis system (Promega, USA) following the instructions in the Promega kit

158 (https://tools.thermofisher.com/content/sfs/manuals/superscriptIII_man.pdf).

159 Quantitative real-time RT-PCR (qRT-PCR) experiments were carried out applying the cDNA,

160 SYBR Green Master mix, the specific primers of cotton PYL genes (Table S1, the primer

161 sequences were submitted to $\mathrm{NCBI}$ ), and an $\mathrm{ABI} 7500$ real-time PCR system. Cotton $U B Q 7$ was

162 used as the internal control. Experiments were repeated at least three times.

163 Yeast two-hybrid assay

164 Full-length sequences of gene GhPYLs and GhABIl were cloned into pGBKT7 and pGADT7

165 vectors, respectively, using primers listed in Table S2 (the primer sequences were submitted to

$166 \mathrm{NCBI).} \mathrm{The} \mathrm{resultant} \mathrm{constructs} \mathrm{were} \mathrm{co-transformed} \mathrm{into} \mathrm{yeast} \mathrm{strain} \mathrm{AH109} \mathrm{(MATa,} \mathrm{trp1-901,}$

167 leu2-3, 112, ura3-52, his3-200, gal4 $\Delta$, gal80 $\Delta$, LYS2: :GAL1UAS-GAL1TATAHIS3, GAL2UAS-

168 GAL2TATA-ADE2, URA3: :MEL1 UASMEL1TATA-lacZ, MEL1) according to the method

169 described in page 18-21 in Yeast Protocols Handbook (Clontech, 
170 http://www.clontech.com/xxclt_searchResults.jsp). The cotransformants were plated on non-

171 selective SD/-Leu/-Trp (synthetic dropout medium without Leu and Trp) agar plates and selective

$172 \mathrm{SD} /$-Leu/-Trp/-His/-Ade agar plates in the absence or presence of ABA. A cotransformed yeast

173 spot was collected and diluted in sterile $\mathrm{ddH}_{2} \mathrm{O}$. The optical density (OD) value for the first

174 solution of yeast colony was set 1 . Serial 1:10 dilutions were generated, and $2 \mu \mathrm{L}$ of the dilution

175 was dropped on an agar plate to obtain one spot. The interactions between GhPYLs and GhABI1

176 were observed after $4 \mathrm{~d}$ of incubation at $30^{\circ} \mathrm{C}$. The experiments were repeated at least three

177 times.

178 RESULTS

179 Genome-wide analysis of PYLs in four cotton species

180 To investigate the PYL family in the cotton genomes, the 14 AtPYL gene coding sequences and

181 amino acid sequences were applied as queries to search against the cotton genome databases. A

182 total of 21, 20, 40 and 39 PYL genes were identified in the genomes of two progenitor diploid

183 species G. arboretum and G. raimondii, and their derived tetraploid species G. hirsutum and $G$.

184 barbadense, respectively. The PYLs in the two diploid species were named based on their

185 orthologous similarity to the 14 AtPYLs according to the methods described by Mohanta et al.

186 (2015) in other genes. Briefly, the first letter of the genus (upper case) and the first letter of the

187 species (lower case) followed by PYL and an AtPYL number were used to name a Gossypium

188 PYL. The number of a Gossypium PYL was the same to that of its orthologous AtPYL sharing the

189 most similarity in protein sequences to the Gossypium PYL. When more than one Gossypium

190 PYLs had the same ortholog in Arabidopsis, additional numbers followed by a hyphen were 
191

192

applied to distinguish among paralogs of the Gossypium PYLs. The small number after the hyphen means high similarity of a cotton PYL to its corresponding AtPYL. The PYLs of the tetraploid cotton plants were denominated based on their phylogenetic relationship with those in G. arboretum and G. raimondii; and the last letter A or D meaned that the PYL was derived from A or D genome (Table S3). Therefore, the Gossypium PYLs in the four species were named GaPYLs, GrPYLs, GhPYLs and GbPYLs, respectively. We noticed that the Gossypium PYLs named following AtPYL2, AtPYL4, AtPYL6, AtPYL9, AtPYL11, AtPYL12, and AtPYR1. Moreover, most of these AtPYLs possessed not only one ortholog in Gossypium. For example, 8, 7, 14 and 13 homologs of AtPYL9 were identified in genomes of G. arboretum, G. raimondii, G. hirsutum and G. barbadense, respectively (Table S3).

Analysis of the physical properties of the Gossypium PYL members revealed that these PYLs were highly conserved. Most PYLs had similar amino acid lengths, molecular weights (MWs), and theoretical isoelectric points (pI). Majority of PYLs in Gossypium possessed 177-222 amino acids. The MWs of the PYLs varied from $15.32 \mathrm{kDa}$ to $32.22 \mathrm{kDa}$. The pIs of PYLs ranged from 4.73 to 9.51 with an average of 6.20. Most PYL proteins were predicted to locate in cytoplasm and/or nucleus (Table S3).

\section{Phylogenetic and structural analysis of cotton PYLs}

To explore the evolutionary relationship of the PYLs among G. arboreum, G. raimondii, G.

hirsutum and G. barbadense, an unrooted phylogenetic tree for the 120 PYLs was generated (Fig.

2A, Files S1-S2.). The PYLs can be divided into three subfamilies (I to III) based on the bootstrap values $(>1000)$ in the Neighbor-Joining (NJ) tree. Subfamily I had 41 members, which were orthologs of AtPYR1 (16) and AtPYL2 (25). Subfamily II consisted of 37 PYL genes. They 
213 were homologs of AtPYL4 (17), AtPYL6 (14), AtPYL11 (3) and AtPYL12 (3). Other 42 PYL

214 members (homologues of AtPYL9) belonged to subfamily III (Fig. 2A). We found that the

215 Gossypium homologs of the same AtPYL frequently clustered closely, indicating their more

216 closed relative relationship. Besides, several PYLs including GaPYL2-2, GrPYL2-2, GhPYL2-

217 2A, GrPYL2-2D, GbPYL2-2A, GbPYL2-2D and GbPYL2-2D' appeared to be distant clades

218 from other PYLs in Gossypium (Fig. 2A). This suggests that these PYL2-2 members had

219 relatively distant phylogenetic relationship with other Gossypium PYLs.

220 The exon/intron structures of the Gossypium PYL genes were studied. The results showed that

221 the number of exons in the PYLs was 1 to 3 . There was no intron in $66 P Y L$ genes. Most of the

222 remaining genes had two introns except that 9 members had one intron (Fig. 2B). In addition,

223 most Gossypium PYL genes clustered in the same subfamily had the similar number of exons and

224 lengths of introns. For instance, vast majority of genes in subfamily I and II had only one exon,

225 and 41 out of 42 genes in subfamily III had 3 exons and relatively long intron sequences (Fig.

2B). These results imply that the exon/intron organizations of cotton PYLs are closely related to

the phylogenetic relationship of the genes.

228 To further clarify the diversity of motif compositions, the putative motifs in the $120 \mathrm{PYL}$

229 proteins in subfamily I to III were analyzed by the MEME software. A total of 19 motifs

230 designated as motif 1 to motif 19 were detected (Fig. 2C). The numbers of motifs in a PYL of

231 subfamily I to III were 4-7, 4-7 and 3-6, respectively. Of the 19 motifs, motif 8 and 10 emerged in

232 majority of PYLs in subfamily I, motif 3 and 5 were conservative among most PYLs in

233 subfamily II; and motif 1, 2, 4 and 9 existed in overwhelming majority of subfamily III members.

234 Most PYLs within the same subfamilies have very similar motif compositions and distributions, 
235 implying that PYLs within the same subfamilies probably share similar functions. Intriguingly,

236 some motifs specifically existed in a particular subfamily or some PYLs. For example, motif 16

237 and 17 were only present in subfamily II, and motif 12 and 18 only belonged to members of

238 subfamily III. Motif 11 was only seen in GbPYL2-3A and GbPYL9-4D. Motif 7 was only found

239 in GhPYL2-4A and GbPYL6-2D'; and motif 15 was only observed in GbPYL6-2D'. These

240 findings suggest that these motifs might play specific roles in the PYLs of that subfamily or in

241 those PYLs. The detailed mechanisms need to be experimentally examined in the future.

\section{Conserved domains and amino acids of cotton PYLs}

243 To better understand the structural similarity of Gossypium PYLs, the amino acid sequences of

244 PYLs from G. arboreum, G. raimondii and G. hirsutum as well as those from the two diploid

245 species and G. barbadense were aligned, respectively (Fig. S1, Fig. S2). The results revealed that

246 these PYLs had high sequence similarities. All the PYLs shared a similar helix-grip structure

247 formed by seven $\beta$-sheets and three $\alpha$-helices, and four identical conserved loops among the $\beta$ -

248 sheets and the $\alpha$-helices (Fig. S1, Fig. S2). Furthermore, many conserved amino acids

249 (highlighted by red colour) were observed in the putative $\beta$-sheets, $\alpha$-helices as well as loops

250 (Fig. S1, Fig. S2), consistent with the structure of AtPYLs in Arabidopsis (Ma et al., 2009; Park

251 et al., 2009). These conserved secondary structures and amino acids have been demonstrated to

252 be essential for the functions of ABA receptors in Arabidopsis. For instance, the loops of CL1,

253 CL2 and CL3 are essential for ABA bindings and the PYL-PP2C interactions (Zhang et al.,

254 2015). Noteworthily, the conserved leucine (L) was replaced by methionine (M) in the CL2 loop,

255 and the conserved arginine (R) was replaced by lysine (K) in the CL3 loop in GaPYL6-2,

256 GbPYL6-2A' and GbPYL6-2D' (Fig. S1, Fig. S2). Similarly, the conservative arginine (R) was 
257 replaced by methionine (M) in the CL3 loop in GhPYL9-5D (Fig. S1). These data hint that these

258 PYLs may be significantly different in ABA bindings and interactions with PP2C from other

259 Gossypium PYLs.

260 Chromosomal distributions of cotton PYLs

261 The localizations of the Gossypium PYLs in chromosomes were determined. Generally, PYL

262 genes were unevenly distributed on multiple chromosomes (Fig. 3). The 21 GaPYLs, 20 GrPYLs,

26340 GhPYLs and 39 GbPYLs separately placed on 10, 11, 21 and 18 chromosomes, respectively.

264 Four genes were located on each of the Achr10and Atchr05 chromosomes. Many chromosomes,

265 each possessed 3 genes. These chromosomes included Achr06 from G. arboretum, Dchr07 and

266 Dchr08 from G. raimondii, Atchr11, Atchr12, Dtchr05, Dtchr11 and Dtchr12 from G. hirsutum;

267 and At'chr01, At'chr10, Dt'chr08, Dt'chr10, Dt'chr11 and Dt'chr12 from G. barbadense (Fig. 3). A

268 large number of chromosomes individually owned two genes. These chromosomes were Achr03,

269 Achr07, Achr09 and Achr13 in G. arboretum, Dchr04, Dchr06 and Dchr11 in G. raimondii,

270 Atchr08, Atchr09, Atchr10, Dtchr08, Dtchr09 and Dtchr10 in G. hirsutum; and At'chr12, Dt

271 'chr05 and Dt'chr09 in G. barbadense. Each of the rest chromosomes had 1 gene. The

272 distributions of the PYL genes on individual chromosome were irregular. Some genes were

273 located on the upper end of the chromosome arms, some placed on the lower end of the arms;

274 whereas some lied in the region far from two end of the arms (Fig. 3). Besides, 2 genes in $G$.

275 arboretum (GaPYL2-1, GaPYL2-3) and 6 genes in G. barbadense (GbPYL4-2A, GbPYL9-3A,

276 GbPYL9-5A, GbPYL9-6D', GbPYL11A and GbPYL12D) were present in scaffolds.

277 We compared the positions of the orthologs among GaPYLs, GrPYLs and GhPYLs in 278 chromosomes. Unexpectedly, only a few PYL homologs localized in their corresponding 
279 homoeologous chromosomes. Similar results also happened among GaPYLs, GrPYLs and

280 GbPYLs (Fig. 3). These findings hint that many complex conversion events of PYL-contained

281 homoeologous chromosomes or of PYLs occurred among different Gossypium species during

282 evolution.

283 Synteny analysis of PYL genes

284 It has addressed that gene duplication events including tandem and segmental duplications play

285 key roles in expanding gene family during the evolutionary process (Cannon et al., 2004). To

286 gain insight into the genetic origins and evolution of the Gossypium PYLs, we analyzed the

287 homologous gene pairs of PYLs among G. arboreum, G. raimondii and G. hirsutum. A sum of 88

288 collinearity blocks were identified between $G$. Arboreum and G. raimondii, and each block had

289 one gene pair. Ninety-one collinearity blocks with 93 homologous pairs were detected between

290 the At-genome and Dt-genome of G. hirsutum. Moreover, two homologous gene pairs between

291 chromosome At11 and Dt11 (GhPYL9-3A/GhPYL9-4D, GhPYR1-2A/GhPYR1-2D), and

292 chromosome At12 and Dt12 (GhPYL9-5A/GhPYL9-5D, GhPYR1-3/GhPYR1-3D) were found in

293 two individual collinearity blocks. Additionally, 395 homologous gene pairs distributed in 385

294 collinearity blocks among G. Arboreum, G. raimondii and G. hirsutum (Fig. 4, Table S4). Of

295 these blocks, one harbored three homologous gene pairs (GrPYL9-1/GhPYL9-1D, GrPYL9-

296 4/GhPYL9-4D, GrPYR1-2/GhPYR1-2D). The block was between chromosome D07 in $G$.

297 raimondii and chromosome Dt11 in G. hirsutum. Other collinearity blocks individually possessed

298 two homologous gene pairs (Table S4). No gene pair was implicated in tandem duplication.

299 These results suggest that segmental duplications dominantly contribute to the generation of

300 Gossypium PYLs during genetic evolution. 


\section{Analysis of Ka/Ks values of PYLs in G. arboreum, G. raimondii and G. hirsutum}

To further investigate the divergence and selection in duplication of $P Y L$ genes, the nonsynonymous $(\mathrm{Ka})$, synonymous $(\mathrm{Ks})$ and $\mathrm{Ka} / K \mathrm{~s}$ values were evaluated for the homologous gene pairs among G. arboreum, G. raimondii and G. hirsutum. The results indicated that the average $\mathrm{Ka} / \mathrm{Ks}$ values among homologous gene pairs of PYLs between genomes and/or subgenomes AA, AtAt, AtDt, DtDt, AD, AAt, ADt, DAt, DDt, DD were 0.31, 0.47, 0.36, 0.31, 0.42, 0.29, 0.34, 0.31 and 0.42 , respectively (Fig. 5). The average $\mathrm{Ka} / \mathrm{Ks}$ value of all the 301 gene pairs was 0.17 , less than 1 . These data suggest that these genes were mainly under the purifying selection during evolution. In contrast, the ratio of Ka to Ks between GhPYL2-4A and GaPYL2-4 was 1.08, and that between GhPYL9-8A and GrPYL9-7 was 1.51, indicating that the two gene pairs may generate under positive selection. Besides, the $\mathrm{Ka} / \mathrm{Ks}$ values of many gene pairs were 1 (Table S5), implying that these genes are under neutral evolution.

\section{Phylogenetic relationship of PYLs in cotton and other plant species}

To determine the evolutionary relationships of PYLs among the four cotton species and other plants, full-length amino acid sequences of the predicted PYLs were downloaded from the databases of G. arboretum, G. raimondii, G. hirsutum, G. barbadense, T. cacao, R. communis, $V$. vinifera, B. distachyon, O. sativa and A. thaliana (Table 1,Files S3-S5.), and a phylogenetic tree was constructed applying the neighbor-joining method and the MEGA 5.0 software. On the basis of the topologic structure, the PYL family members were classified into three subfamilies (I-III)

(Fig. 6). Expectedly, vast majority of the Gossypium PYLs were closely clustered in a subfamily. Moreover, every subfamily contained PYLs from eudicots such as Gossypium, cocoa, castor and Arabidopsis, and from monocots like $B$. distachyon and $O$. sativa, hinting that these PYLs 
323 generated before the divergence from eudicots and monocots. In addition, numerous PYLs from

324 cotton species clustered more closely with those from cocoa than from other plants (Fig. 6),

325 reflecting closer relationship of Gossypium PYLs with cacao PYLs relative to other plant PYLs.

326 Expression of GhPYL genes in tissues

327 To further understand the roles of cotton PYLs in diverse organs, the expression patterns of all

328 the 40 GhPYL genes were monitored by qRT-PCR. Of these GhPYLs, 22 genes were

329 preferentially expressed in the flower, 10 genes were dominantly expressed in the root. Moreover,

3303 genes including GhPYR1-3D, GhPYL2-2A and GhPYL2-2D were highly expressed in the fiber.

331 The transcripts of GhPYR1-3A, GhPYL4-3A, GhPYL4-3D, GhPYL9-1A and GhPYL12D were

332 relatively abundant in the stem (Fig. 7). These results indicate that some ABA receptors may

333 specially function in a unique tissue. Besides, more GhPYLs were predominantly expressed in the

334 flower and the root, suggesting that ABA receptors may play important roles in fiber formation

335 and in response to abiotic stresses in cotton.

\section{Expression profiles of GhPYLs in responses to ABA and osmotic stress}

337 The expression patterns of the GhPYL genes in roots were monitored after treatments with 100

$338 \mu \mathrm{M}$ ABA or 10\% PEG6000 for different period of time. In general, the transcriptional levels of

339 most GhPYLs were decreased in responding to ABA (Fig. 8), and moderately enhanced in

340 response to osmotic stress (Fig. 9). After treatment with ABA for 3 h or 6 h, expression of many

341 genes for example GhPYR1-1A, GhPYR1-3D, GhPYL9-5A significantly reduced, then increased

342 slowly at $12 \mathrm{~h}$ or $24 \mathrm{~h}$. Expression of some genes like GhPYL4-1D, GhPYL4-2A and GhPYL6-1D

343 continually decreased in response to ABA. However, the transcriptional abundances of some

344 genes increased upon ABA treatment. These genes included GhPYL2-2A, GhPYL2-3A, GhPYL2- 
345

346

347

348

349

350

351

3D, GhPYL4-3D, GhPYL6-2A, GhPY6-2D, GhPYL9-1D and GhPYL12D. By contrast, the expression of GhPYL9-2D was not influenced by ABA treatment (Fig. 8). These results indicate that GhPYLs play differential roles in perceiving ABA signals.

The expression of most GhPYLs was upregulated by PEG treatments for $12 \mathrm{~h}$ and/or $24 \mathrm{~h}$. By contrast, the transcriptional levels of GhPYR1-3, GhPYR1-3D, GhPYL2-1A and GhPYL2-4D were diminished whereas those of GhPYRl-2A and GhPYRl-2D were unchanged after exposure to high concentration of PEG6000 (Fig. 9). These results suggest that a great number GhPYLs have different responses to ABA relative to osmotic stress in cotton.

\section{Many GhPYLs interact with GhABI1A or GhABI1D}

PYLs have been addressed to transduce ABA signals to downstream targets through selectively interplaying with clade A PP2C proteins such as ABI1 and ABI2 in plants (Cutler et al., 2010; Joshi-Saha et al., 2011). We therefore examined the interactions between GhPYLs and GhABI1A (Gh_A07G0123) or GhABI1D (Gh_D07G2383) in the absence or presence of ABA by yeast-two hybrid method. Twenty-five out of fourty GhPYLs were cloned because of high similarity of CDS sequences among different GhPYL members. In the absence of ABA, 9 GhPYLs individully interplayed with GhABI1A, and 8 GhPYLs respectively interacted with GhABI1D (Table 2).

Among them, GhPYR1-1D, GhPYL6-2D, GhPYL9-1A and GhPYL9-5D displayed relative weak interactive signals with GhABI1D. GhPYL6-2A and GhPYL9-4D showed strong interaction signals with GhABI1D (Fig. 10). When supplied with $10 \mu \mathrm{M}$ in the medium, 17 GhYPLs could interact with GhABI1A, and 14 GhPYLs could interplay with GhABI1D. We observed that many interactions were ABA-dependent, and numerous interactions were ABA-independent (Fig. 10, Table 2). Moreover, the interactive intensities between GhPYL6-2A and GhABI1A as well as 
between GhPYL9-5D and GhABI1D were increased by ABA. Interestingly, the interaction signal between GhPYL4-2A and GhABI1A was slightly weakened by ABA. These results suggest that

GhPYLs differentially or specifically bind to GhABI1 in response to stresses, reflecting the diverse interacting modes among GhPYLs, GhABI1 and ABA in cotton.

\section{1}

372

\section{DISCUSSION}

ABA receptor PYLs are key regulators of ABA signaling, and have been investigated in many plants in recent years (Boneh et al., 2012; Kim et al., 2012; Bai et al., 2013; González-Guzmán et al., 2014; Fan et al., 2016; Gordon et al., 2016; Zhang et al., 2016; Chen et al., 2017; Guo et al., 2017; Liang et al., 2017). However, knowledge about phylogenesis and roles of most PYLs in Gossypium is limited. In this report, we identified 21, 20, 40 and 39 PYL genes in genomes of $G$. Arboreum, G. raimondii, G. hirsutum and G. barbadense, respectively (Table S3). Compared with the number of PYLs in the reported plants including an Arabidopsis (14), a rice (13), a barley (9), a maize (11), a tomato (15), a Brachypodium distachyon (12), a soybean (23), a poplar (14) and a rubber tree (14), that in Gossypium especially in the tetraploid species was very large (Kim et al., 2012; Bai et al., 2013; González-Guzmán et al., 2014; He et al., 2014; Seiler et al., 2014; Fan et al., 2016; Yu et al., 2016; Zhang et al., 2017; Guo et al., 2017), indicating more complex ABA responses likely happen in Gossypium. Chen et al. (2017) identified 27 GhPYLs (Chen et al., 2017), significantly less than 40 members as described in this study. The main reason may be that the genome sequence database of $G$. hirsutum they chose to search homologs of AtPYLs was different from what we did. Chen et al. (2017) used the database from the website http://cgp.genomics.org.cn/page/species/index.jsp whereas we applied that from https://www.cottongen.org. Liang et al. (2017) reported that there exist 22, 22, 44 and 36 PYLs in 
G. arboretum, G. raimondii, G. hirsutum and G. barbadense, respectively, inconsistent with our results. The possible reason might be the standard we used to determine PYLs in Gossypium was slightly different from what they did. For instance, we also surveyed 44 putative GhPYLs. However, three of them (Gh_A10G0340, Gh_D10G0346 and Gh_D08G1226) lack the PYR_PYL_RCAR_like domain of PYLs, and Gh_Sca112916G01 encodes a too small protein. Accordingly, the four proteins were eliminated in this report. every member had its corresponding ortholog in $a$ G. arboretum and a G. raimondii. Likewise, most GbPYLs individually possessed their corresponding orthologs in a G. arboretum and a $G$. raimondii. However, both GbPYL6-2A and GbPYL6-2D had high sequence similarity to GrPYL6-2. Similar cases occurred in GbPYL6-2A' and GbPYL6-2D', GbPYL2-2D and GbPYL2$2 D^{\prime}$ as well as in GbPYL9-6D and GbPYL9-6D'. The three pairs of genes seemed to derived from GaPYL6-2, GrPYL2-2 and GrPYL9-6, respectively (Table S3). These findings suggest that gene replication events contribute to the generation of these GbPYL pairs in sea island cotton. Moreover, GaPYL9-4 had no corresponding homologous gene in GhPYLs, and some GaPYLs (GaPYR1-1, GaPYR1-2, GaPYL9-1, GaPYL9-2, GaPYL9-4) and GrPYL4-2 did not share corresponding homologous genes in GbPYLs (Table S3), implying that gene loss events occurred during the evolution processes of Gossypium PYLs.

$$
\text { We observed that some AtPYLs (for example AtPYL9) had several homologues of GaPYLs, }
$$
GrPYLs, GhPYLs or GbPYLs (Table S3), hinting that selective gene replications likely play essential roles in the generation of the Gossypium PYLs during evolution. The PYLs of G. arboreum and G. raimondii exhibited similar physical properties to those of 
411 G. hirsutum and G. barbadense (Table S3). This suggests that the functions of the PYLs in the

412 four cotton plants were conserved during the processes of genetic evolution. The predicted

413 subcellular localizations of PYLs in Gossypium were cytoplasm and/or nucleus, in line with the

414 locations of PYLs from Arabidopsis, rice, and soybean plants (Ma et al., 2009; Bai et al., 2013;

415 Tian et al., 2015). These data indicate that the functions of PYLs are conservative among

416 different plant species.

417 We saw that each subfamily of Gossypium PYLs contained several members of G. arboreum,

G. raimondii, G. hirsutum and G. barbadense. Moreover, the number of GhPYLs and GbPYLs

was roughly sum of GaPYLs and GrPYLs. These findings imply that each diploid species already

contained about 20 PYL members prior to formulating $G$. hirsutum and G. barbadense, and the

GhPYLs and GbPYLs mainly came from the allopolyploidization (Wendel et al., 2010).

The organizations of intron/exon and the number of exons in the surveyed Gossypium PYLs

were very similar to those in the orthologs in rice, maize, tomato, rubber tree and Brachypodium

distachyon (González-Guzmán et al., 2014; He et al., 2014; Fan et al., 2016; Guo et al., 2017;

Zhang et al., 2017). Moreover, all of PYLs in Gossypium had similar helix-grip structures

consisting of seven $\beta$-sheets, three $\alpha$-helices and four loops, consistent with PYLs in Arabidopsis,

rice and other plant species (Ma et al., 2009; González-Guzmán et al., 2014; He et al., 2014; Fan

et al., 2016; Guo et al., 2017; Zhang et al., 2017). These results hint that these PYLs from

different plant species have very similar functions and action modes.

430 Most Gossypium PYLs in subfamily I and II had no intron. However, some members in the two 
433 Colinearity results revealed that approximate 400 homologous gene pairs existed among PYLs

434 from G. arboreum, G. raimondii and G. hirsutum (Fig. 4), and numerous PYL gene pairs like

435 GbPYL2-2D and GbPYL2-2D' had nearly identical amino acid sequences (Table S6). These

436 results suggest that PYL family expands through segmental duplication events during evolution.

437 To uncover the homologous relationships of PYL gene family among different taxa, a

438 phylogenetic tree was constructed based on the PYL protein sequences from four cotton species,

439 T. cacao, R. communis, V. vinifera, B. distachyon, rice and Arabidopsis (Fig. 6). We observed

440 Gossypium PYLs clustered more closely with cocoa PYLs than OsPYLs, in agreement with the

441 evolutionary relationships among these plants.

442 Expression analysis results revealed that great majority of GhPYLs were expressed in various

443 tissues like roots, stems, leaves, flowers and fibers. Notably, a large number of genes were highly

444 expressed in the flower (Fig. 7). These results imply that ABA is implicated in modulation of

445 reproductive development in cotton. Moreover, we noticed that some genes were preferentially

446 expressed in roots, stems and fiber, pointing to the important roles of ABA in these tissues. PYLs

447 have been documented to express in diverse tissues in various plants such as rice, maize and

448 rubber tree (Tian et al., 2015; Fan et al., 2016; Guo et al., 2017). In rice, most OsPYLs were

449 expressed in all tissues, $O S P Y L 3$ and $O S P Y L 5$ were predominantly expressed in leaves, and

450 OsPYL1 in roots (Tian et al., 2015). In maize, PYL11 was upregulated in leaves, PYL6 and

451 PYL10 in roots (Fan et al., 2016). In rubber tree, five genes were detected to express in all tissues

452 tested, four genes were preferentially expressed in leaves, four in roots and one in flowers (Guo

453 et al., 2017). These results were consistent with our findings in cotton, indicating the diverse

454 biological functions of different PYLs in plants. 
455 Changes in the expression levels of 40 GhPYLs were investigated in roots in responding to

456 ABA or osmotic stress. The transcriptional abundances of many genes decreased after ABA

457 treatments (Fig. 8), but increased post PEG treatments (Fig. 9), pointing to the different

458 functional mechanisms of GhPYLs in response to ABA and osmotic stress. Tian et al. (2005)

459 reported OsPYLs are differentially expressed after ABA treatment. Some AtPYLs have been

460 addressed to be down-regulated by ABA treatment in Arabidopsis (Santiago et al., 2015). In B.

461 distachyon, BdPYL11 is down-regulated under ABA and PEG6000 treatments while BdPP2CA4,

462 BdPP2CA5, BdPP2CA6 and BdPP2CA8 are up-regulated (Zhang et al., 2017). ZmPYL4-11 was

463 found to be down-regulated by ABA (Fan et al., 2016). Bai et al. (2013) demonstrated that

464 expression of many PYLs is reduced while the transcriptional abundances of some genes are

465 enhanced upon ABA treatment in soybean. Together, these results indicate different PYL

466 members have differential expression patterns in responding to ABA or osmotic stress, reflecting

467 the diversity of PYLs in plants.

468 The interactions between 25 GhPYL members and GhABI1A or GhABI1D were analyzed by

469 two-yeast hybrid method in the absence or presence of ABA. The results showed that GhPYLs

470 selectively and specifically interacted with the two GhABI1s. Furthermore, these interactions

471 were ABA-dependent or ABA-independent (Fig. 10). These results suggest that the GhPYLs

472 should be functional ABA receptors in cotton. In Arabidopsis, AtPYL6, and AtPYL9 inhibit

473 PP2Cs in a ABA-independent manner (Hao et al., 2011; Miyakawa et al., 2013). Consistently, a

474 number of homologs of AtPYL6 and AtPYL9 such as GhPYL6-2D, GhPYL9-4D and Gh9-6A

475 interacted with GhABI1A or GhABI1D without ABA, implying the conserved mechanism of

476 ABA receptors between Arabidopsis and cotton. However, some cotton homologs of AtPYL4 
477 (GhPYL4-2A) could interact with GhABIs in the absence of ABA whereas some other homologs

478 of AtPYL4 (GhPYL4-1A, GhPYL4-2D) interplayed with GhABIs relying on ABA, although

479 AtPYL4 suppresses PP2Cs without ABA (Hao et al., 2011; Miyakawa et al., 2013). These data

480 suggest that the action modes of some ABA recepors of cotton may be altered during evolution.

481 The detailed mechanisms need to study in the future.

482 CONCLUSIONS

483 A total of 21, 20, 40 and 39 PYL genes were identified from G. arboretum, G. raimondii, G.

484 hirsutum and G. barbadense, respectively. High commonality of gene structure, amino acid

485 sequences and synteny of PYL members were found among the four surveyed Gossypium

486 species. Specific expression patterns in tissues and diverse expression profiles in response to

487 ABA and osmotic stress were uncovered. The interactions between 25 GhPYLs and 2 GhABI

488 proteins were also investigated. These results suggest that the Gossypium PYLs are diverse

489 functional ABA receptors. Our results gave a comprehensive information of the Gossypium PYLs

490 for further research towards understanding the roles of PYLs in cotton.

\section{REFERENCES}

492 Allen RD. 2010. Opportunities for engineering abiotic stress tolerance in cotton plants. Cotton.

493 Berlin:Springer Verlag, 127-160.

494 Antoni R, Gonzalez-Guzman M, Rodriguez L, Peirats-Llobet M, Pizzio GA, Fernandez

495 MA, De Winne N, De Jaeger G, Dietrich D, Bennett MJ, Rodriguez PL. 2013.

496 PYRABACTIN RESISTANCE1-LIKE8 plays an important role for the regulation of

497 abscisic acid signaling in root. Plant Physiology 161:931-941 DOI

$498 \quad 10.1104 / \mathrm{pp} / .112 .208678$. 
499

500

501

502

503

504

Bai G, Yang DH, Zhao Y, Ha S, Yang F, Ma J, Gao XS, Wang ZM, Zhu JK. 2013. Interactions between soybean $\mathrm{ABA}$ receptors and type $2 \mathrm{C}$ protein phosphatases. Plant Molecular Biology 83:651-664 DOI 10.1007/s11103-013-0114-4.

Boneh U, Biton I, Zheng C, Schwartz A, Ben-Ari G. 2012. Characterization of potential ABA receptors in Vitis vinifera. Plant Cell Reports 31:311-321 DOI 10.1007/s00299-011-1166-z.

Cannon SB, Mitra A, Baumgarten A, Young ND, May G. 2004. The roles of segmental and tandem gene duplication in the evolution of large gene families in Arabidopsis thaliana. BMC Plant Biology 4:10 DOI10.1186/1471-2229-4-10.

Chen Y, Feng L, Wei N, Liu ZH, Hu S, Li XB. 2017. Overexpression of cotton $P Y L$ genes in Arabidopsis enhances the transgenic plant tolerance to drought stress. Plant Physiology \& Biochemistry 115:229-238.

Cutler SR, Rodriguez PL, Finkelstein RR, Abrams SR. 2010. Abscisic acid: emergence of a core signaling network. Annual Reviews of Plant Biology 61:651-679 DOI 10.1146/annurev-arplant-042809-112122.

Fan W, Zhao M, Li S, Bai X, Li J, Mu Z. 2016. Contrasting transcriptional responses of PYR1/PYL/RCAR ABA receptors to ABA or dehydration stress between maize seedling leaves and roots. BMC Plant Biology 16:99 DOI 10.1186/12870-016-0764-x.

Fuchs S, Tischer SV, Wunschel C, Christmann A, Grill E. 2014. Abscisic acid sensor RCAR7/PYL13, specific regulator of protein phosphatase coreceptors. Proceedings of the National Academy of Sciences 111:5741-5746 DOI 10.1073/pnas.1322085111.

Fujii H, Chinnusamy V, Rodrigues A, Rubio S, Antoni R, Park SY, Cutler SR, Sheen J, Rodriguez PL, Zhu JK. 2009. In vitro reconstitution of an abscisic acid signalling 
pathway. Nature 462:660-664 DOI 10.1038/nature08599.

Gonzalez-Guzman M, Pizzio GA, Antoni R, Vera-Sirera F, Merilo E, Bassel GW, Fernandez MA, Holdsworth MJ, Perez-Amador MA, Kollist H, Rodriguez PL. 2012. Arabidopsis PYR/PYL/RCAR receptors play a major role in quantitative regulation of stomatal aperture and transcriptional response to abscisic acid. Plant Cell 24:2483-2496 DOI 10.1105/tpc.112.098574.

González-Guzmán M, Rodríguez L, Lorenzo-Orts L, Pons C, Srrión-Perdigones A,

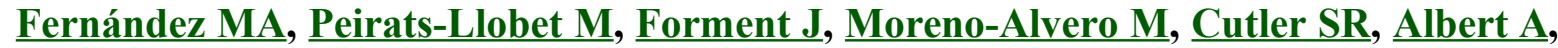
Granell A, Rodríguez PL. 2014. Tomato PYR/PYL/RCAR abscisic acid receptors show high expression in root, differential sensitivity to the abscisic acid agonist quinabactin, and the capability to enhance plant drought resistance. Journal of Experimental Botany 65:4451-4464 DOI 10.1093/jxb/eru 219.

Gordon CS, Rajagopalan N, Risseeuw EP, Surpin M, Ball FJ, Barber CJ, Buhrow LM, Clark SM, Page JE, Todd CD, Abrams SR, Loewen MC. 2016. Characterization of Triticum aestivum abscisic acid receptors and a possible role for these in mediating fusairum head blight susceptibility in wheat. PLoS ONE 11:e0164996 DOI 10.1371/journal.pone.0164996.

Guo D, Zhou Y, Li HL, Zhu JH, Wang Y, Chen XT, Peng SQ. 2017. Identificationand characterization of the abscisic acid (ABA) receptor gene family and its expression in response to hormones in the rubber tree. Scientific Reports 7:45157 DOI $10.1038 /$ srep45157.

Hao Q, Yin P, Li W, Wang L, Yan C, Lin Z, Wang J, Yan SF, Yan N. 2011. Themolecular basis 
of ABA-independent inhibition of PP2Cs by a subclass of PYL proteins. Molecular Cell 42:662-672 DOI 10.1016/j.molcel.2011.05.011.

He Y, Hao Q, Li W, Yan C, Yan N, Yin P. 2014. Identification and characterization of ABA

Kim H, Lee K, Hwang H, Bhatnagar N, Kim DY, Yoon IS, Byun MO, Kim ST, Jung KH, Kim BG. 2014. Overexpression of PYL5 in rice enhances drought tolerance, inhibits growth, and modulates gene expression. Journal of Experimental Botany 65:453-464 DOI 10.1093/jxb/err338.

Larkin MA, Blackshields G, Brown NP, Chenna R, McGettigan PA, McWilliam H, Valentin F, Wallace IM, Wilm A, Lopez R, Thompson JD, Gibson TJ, Higgins DG. 2007. Clustal $\mathrm{W}$ and Clustal $\mathrm{X}$ version 2.0. Bioinformatics 23:2947-2948

DOI 10.1093/bioinformatics/btm404.

Lee HY, Jang G, Um T, Kim JK, Lee JS, Choi YD. 2015. The soluble ABA receptor PYL8 regulates drought resistance by controlling ABA signaling in Arabidopsis. Plant Biotechnology Reports 9:319-330 DOI 10.1007/s11816-015-0366-3.

Lee SC, Luan S. 2012. ABA signal transduction at the crossroad of biotic and abiotic stress 
565

566 567 568 569 570 571 572 573

responses. Plant Cell Environment 35:53-60 DOI 10.1111/j.1365-3040.2011.02426.x.

Liang C, Liu Y, Li Y, Meng Z, Yan R, Zhu T, Wang Y, Kang S, Abid MA, Malik W, Sun G, Guo S, Zhang R. 2017. Activation of ABA receptors gene GhPYL9-11A is positively correlated with cotton drought tolerance in transgenic Arabidopsis. Frontiers in Plant Science 08:1453 DOI10.3389/fpls.2017.01453.

Li W, Wang L, Sheng X, Yan C, Zhou R, Hang J, Yin P, Yan N. 2013. Molecular basis for the selective and ABA-independent inhibition of PP2CA by PYL13. Cell Research 23:13691379 DOI 10.1038/cr.2013.143.

Lim CW, Baek W, Lim S, Lee SC. 2012. ABA signal transduction from ABA receptors to ion channels. Genes \& Genomics 34:345-353 DOI 10.1007/s13258-012-0081-1.

Ma Y, Szostkiewicz I, Korte A, Moes D, Yang Y, Christmann A, Grill E. 2009. Regulators of PP2C phosphatase activity function as abscisic acid sensors. Science 324:1064-8 DOI 10.1126/science. 1172408 .

Miyakawa T, Fujita Y, Yamaguchi-Shinozaki K, Tanokura M. 2013. Structure and function of abscisic acid receptors. Trends in Plant Science 18:259-266 DOI 10.1016/j.tplants.2012.11.002.

Mohanta TK, Mohanta N, Mohanta YK, Parida P, Bae H. 2015. Genome-wide identification of calcineurin B-Like (CBL) gene family of plants reveals novel conserved motifs and evolutionary aspects in calcium signaling events. BMC Plant Biology 15:189 DOI 10.1186/s12870-015-0543-0.

Murashige T, Skoog F. 1962. A revised medium for rapid growth and bio-assays with tobacco tissue culture. Physiologia Plantarum 15:473-497. 
587

588

589

590

591

592

593

594

595

596

Park SY, Fung P, Nishimura N, Jensen DR, Fujii H, Zhao Y, Lumba S, Santiago J, Rodrigues A, Chow TF, Alfred SE, Bonetta D, Finkelstein R, Provart NJ, Desveaux D, Rodriquez PL, McCourt P, Zhu JK, Schroeder JI, Volkman BF, Cutler SR. 2009. Abscisic acid inhibits type 2C protein phosphatases via thePYR/PYL family of START proteins. Science 324:1068-71 DOI 10.1126/science.1173041.

Saavedra X, Modrego A, Rodríguez D, González-García MP, Sanz L, Nicolás G, Lorenzo O. 2010. The nuclear interactor PYL8/RCAR3 of Fagussylvatica FsPP2C1 is a positive regulator of abscisic acid signaling in seeds and stress. Plant Physiology 152:133-150 DOI 10.1104/pp.109.146381.

Santiago J, Rodrigues A, Saez A, Rubio S, Antoni R, Dupeux F, Park SY, Márquez JA, Cutler SR, Rodriguez PL. 2009. Modulation of drought resistance by the abscisic acid receptor PYL5 through inhibition of clade A PP2Cs. Plant Journal 60:575-588 DOI 10.1111/j.1365-313X.2009.03981.x.

Santiago J, Dupeux F, Betz K, Antoni R, Gonzalez-Guzman M, Rodriquez L, Marquez JA, Rodriquez PL. 2012. Structural insights into PYR/PYL/RCARABA receptors and PP2Cs. Plant Science 182:3-11 DOI 10.1016/j.plantsci.2010.11.014.

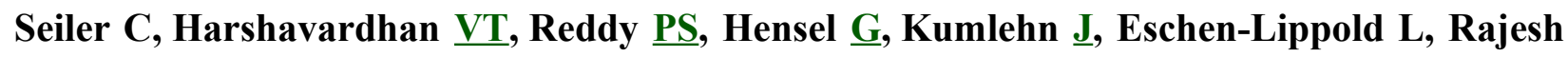
K, Korzun V, Wobus U, Lee J, Selvaraj G, Sreenivasulu N. 2014. Abscisic acid flux alterations result in differential abscisic acid signaling responses and impact assimilation efficiency in barley underterminal drought stress. Plant Physiology 164:1677-1696 DOI 10.1104/pp.113.229062.

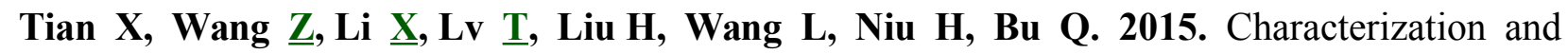


609

610

611

612

613

614

615

616

617

618

619

620

621

622

623

624

625

626

627

628

629

630

functional analysis of pyrabactin resistance-like abscisic acid receptor family in rice. Rice 8:28 DOI 10.1186/s12284-015-0061-6.

Wendel JF, Brubaker CL, Seelanan T. 2010. The origin and evolution of Gossypium. Physiology of cotton. Springer Netherlands:1-18 DOI 10.1007/978-90-481-3195-2_1.

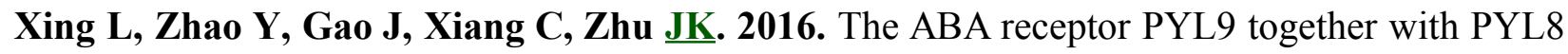
plays an important role in regulating lateral root growth. Scientific Reports. 6:27177 DOI $10.1038 / \operatorname{srep} 27177$.

Yu J, Yang L, Liu X, Tang R, Wang Y, Ge H, Wu M, Zhang J, Zhao F, Luan S, Lan W. 2016. Overexpression of poplar pyrabactin resistance-like abscisic acid receptors promotes abscisic acid sensitivity and drought resistance in transgenic Arabidopsis. PLoS ONE 11:e0168040 DOI 10.1371/journal.pone.0168040.

Zhang F, Wei Q, Shi J, Jin X, He Y, Zhang Y, Luo Q, Wang Y, Chang J, Yang G, He G. 2017. Brachypodiumdistachyon BdPP2CA6 interacts with BdPYLs and BdSnRK2 and positively regulates salt tolerance in transgenic Arabidopsis. Frontiers in Plant Science 8:264 DOI 10.3389/fpls.2017.00264.

Zhang XL, Jiang L, Xin Q, Liu Y, Tan JX, Chen ZZ. 2015. Structural basis and functions of abscisic acid receptors PYLs. Frontiers in Plant Science 6:8 DOI 10.3389/fpls.2015.00088.

Zhao Y, Chan Z, Gao J, Xing L, Cao M, Yu C, Hu Y, You J, Shi H, Zhu Y, Gong Y, Mu Z, Wang H, Deng X, Wang P, Bressan R, Zhu JK. 2016. ABA receptor PYL9 promotes drought resistance and leaf senescence. PNAS 113:1949-1954 DOI $10.1073 /$ pnas. 1522840113.

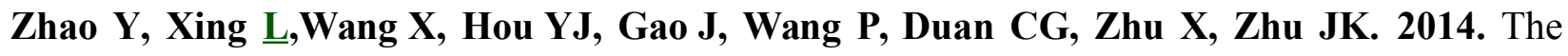


631 ABA receptor PYL8 promotes lateral root growth by enhancing MYB77-dependent 632 transcription of auxin-responsive genes. Science Signaling 7:ra53 DOI $633 \quad 10.1126 /$ scisignal.2005051.

\section{Acknowledgements}

635 The authors thank Dr. Zhen Zhang in Institute of Cotton Research in Chinese Academy of 636 Agricultural Sciences for providing good suggestions. 


\section{Figure 1}

A model showing the ABA key signal transduction pathway.

Arrows indicate positive regulation, bars indicate negative regulation, and $\mathrm{P}$ means phosphorylation. 


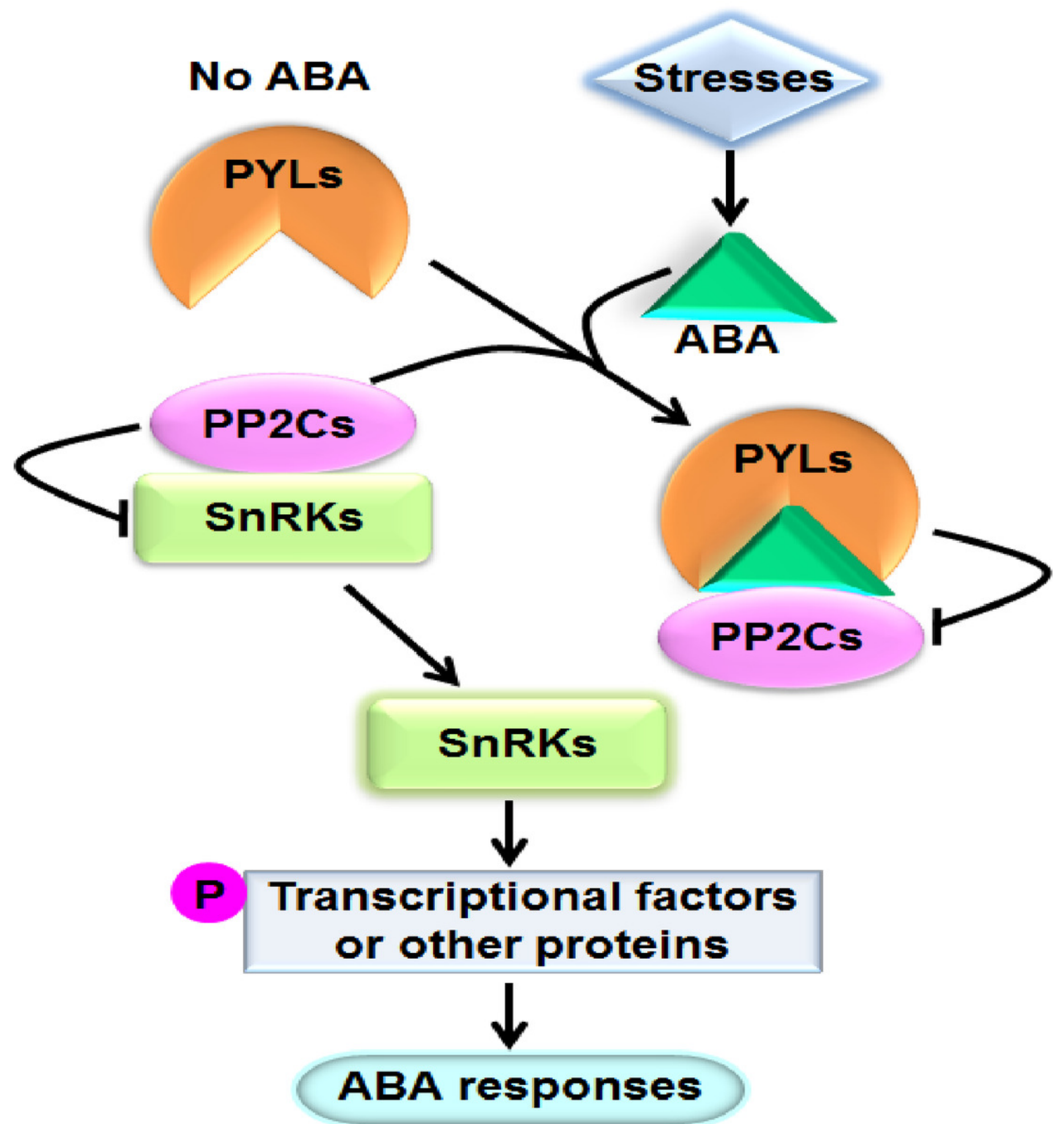




\section{Figure 2}

Phylogenetic relationships, gene architectures and conserved motifs of PYL genes in Gossypium.

( A ) The phylogenetic tree was constructed by the Neighbor-Joining (NJ) method, with 1000 bootstrap replicates. The dark red, pink and green lines show the subfamily I, II and III, respectively. ( B ) Exon/intron architectures of Gossypium PYL genes. The color boxes indicate exons, and the color lines represent introns. The sizes of exons and introns can be calculated following the scale at the bottom. (C) Distributions of conserved motifs. The motifs are indicated by 19 different color boxes. 


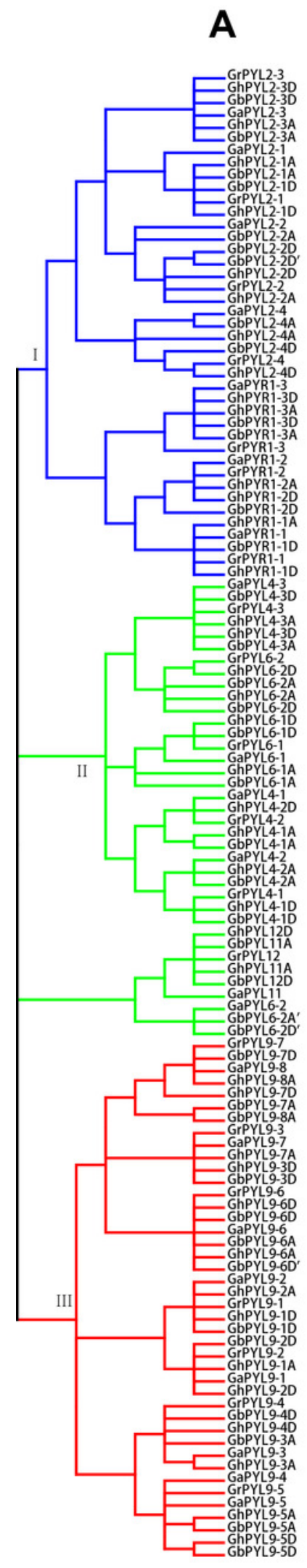

A

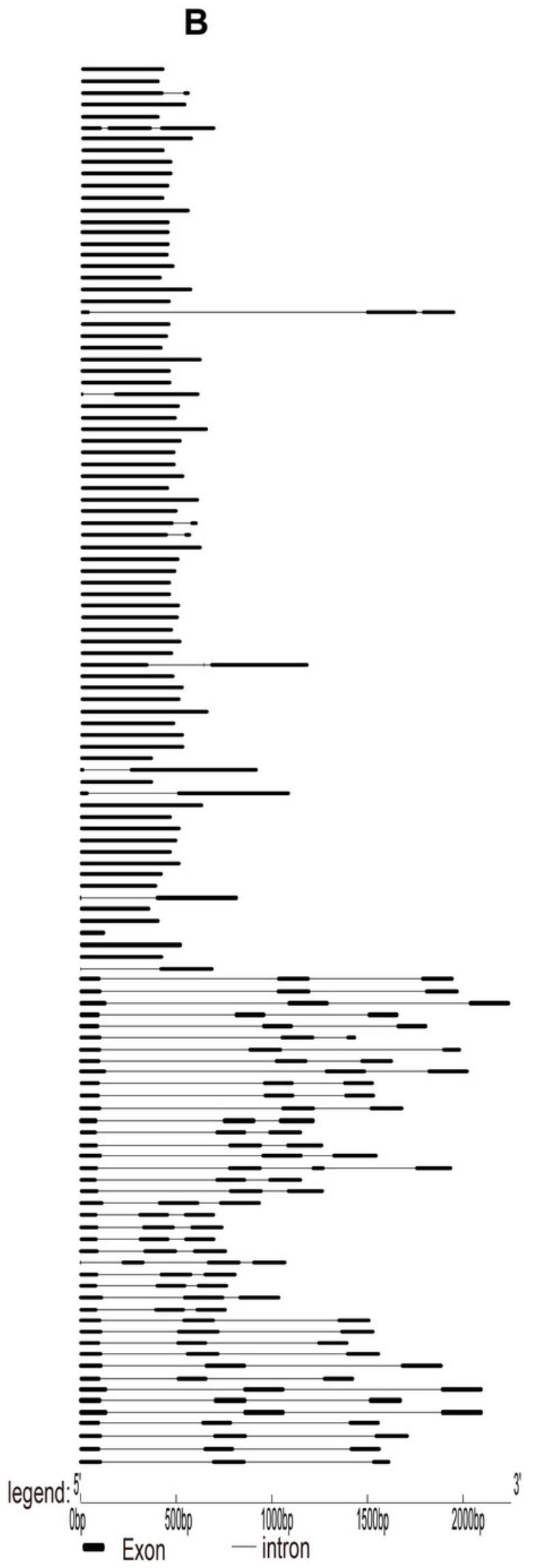

C

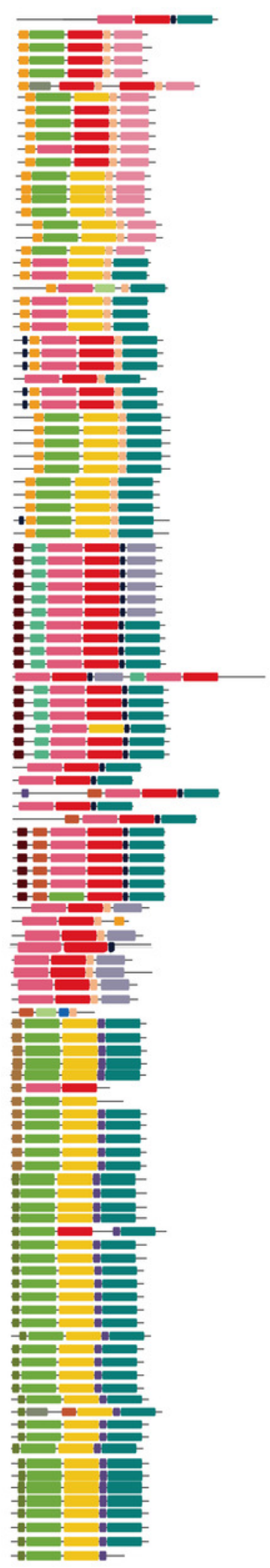

-Motif1 - Motif2 atif3 -Motif4 -Motif5 -Motif6 - Motif $7-$ Motif8 -Motifg - Motif 10 - Motif 11 -Motif 12 - Motif 13 -Motif 14 - Motif 15

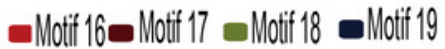


Figure 3

Distributions of Gossypium PYL genes on chromosomes.

GaPYLs, GrPYLs, GhCBLs and GbPYLs were from G. arboreum, G. raimondii, G. hirsutum and G. barbadense, respectively. 

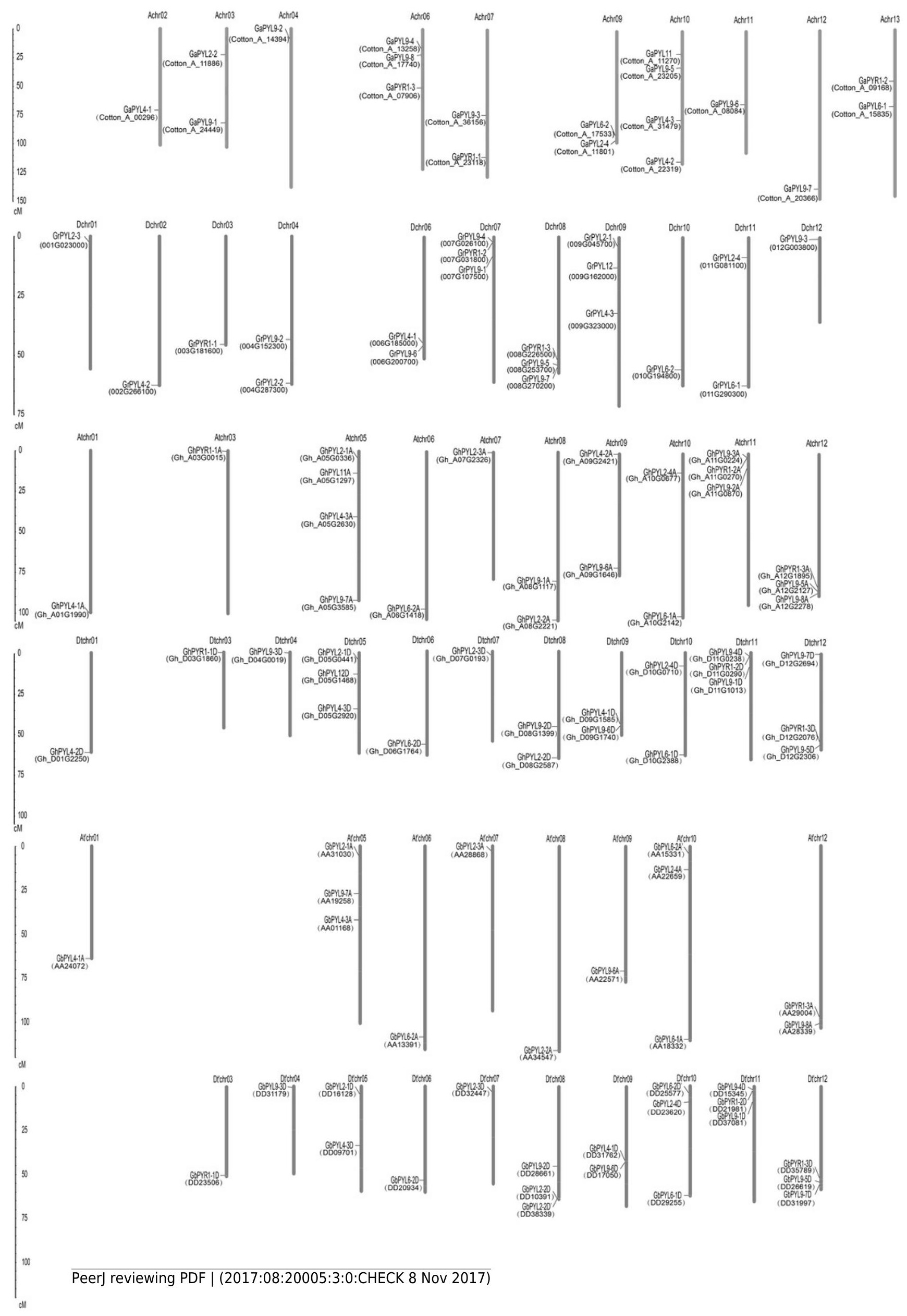


\section{Figure 4}

Genome-wide synteny analysis of PYL genes from G. arboreum, G. raimondii and G. hirsutum.

Synteny analysis between $G$. hirsutum and two diploid species $G$. arboreum and $G$. raimondii. Blue lines link gene pairs between $G$. arboreum and $G$. hirsutum, red lines connect gene pairs between $G$. raimondii and $G$. hirsutum, brown lines bridge gene pairs between $G$. arboreum and $G$. raimondii, black lines join gene pairs in $G$. arboreum, yellow lines link gene pairs in G. raimondii, and green lines connect gene pairs in $G$. hirsutum. 


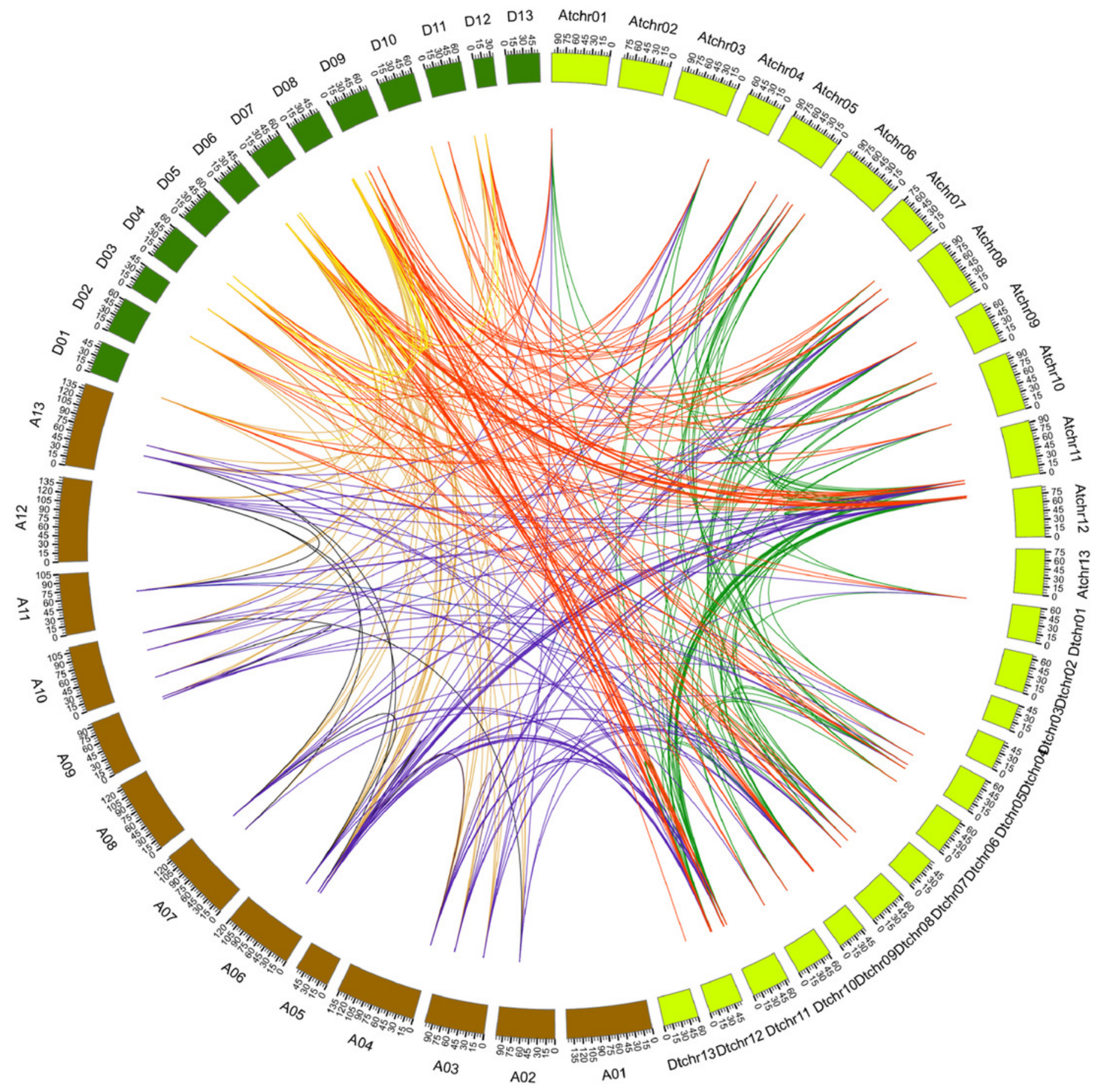


Figure 5

The Ka/Ks values of the homologous PYL gene pairs among A genome of $G$. arboretum (A), D genome of $G$. raimondii (D), At and Dt subgenomes of G. hirsutum ( At, Dt ).

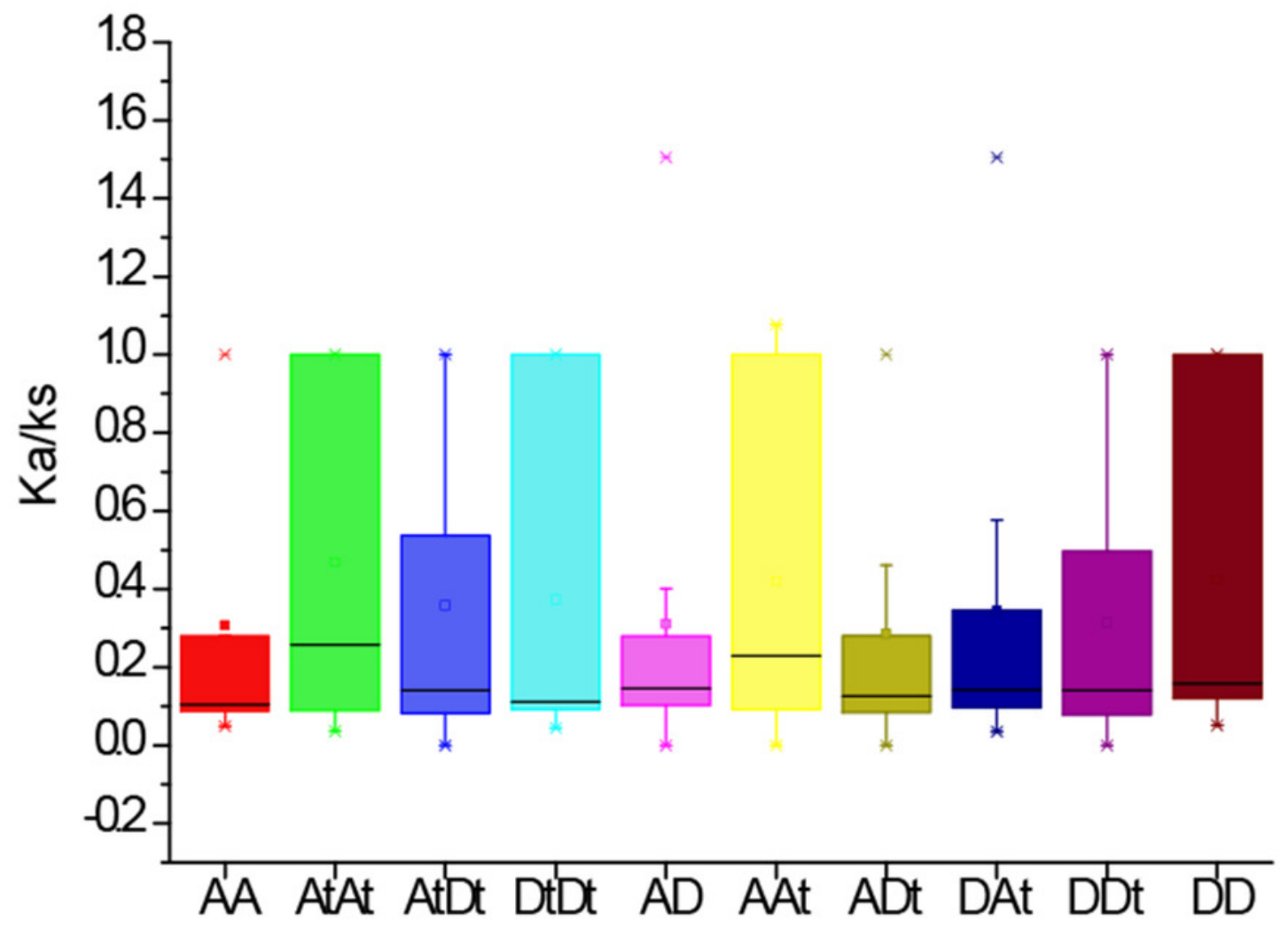




\section{Figure 6}

Phylogenetic tree of PYLs in cotton and other plant species.

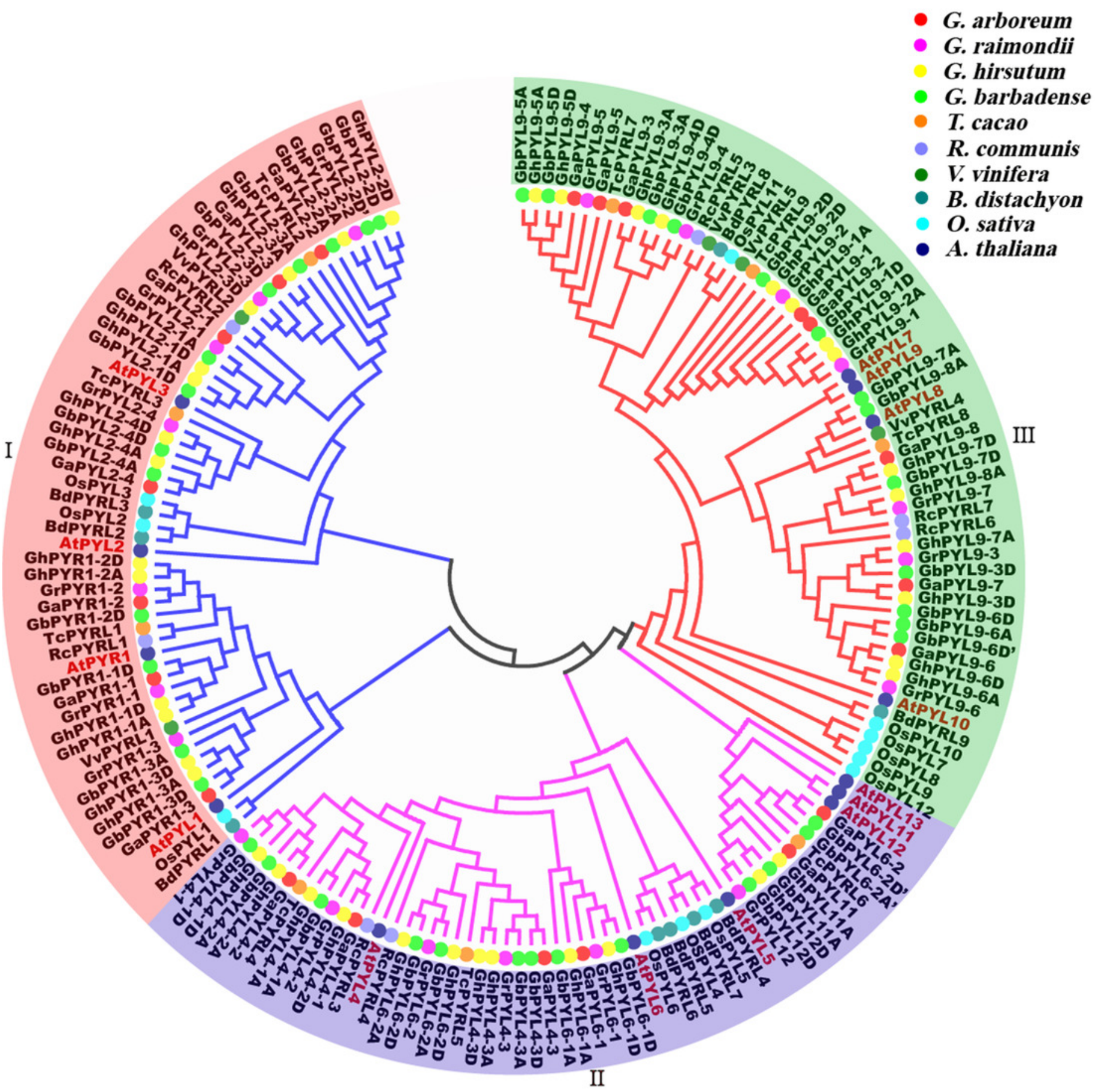


Figure 7

Expression of GhPYL genes in tissues of cotton.

The genes preferentially expressed in flowers (A), roots (B), stems (C) and fibers (D) are shown. Gene GhUBQ7 was used as the internal control. The expression value of a gene in roots was set as 1 . The data are mean $\pm \mathrm{SE}$. 

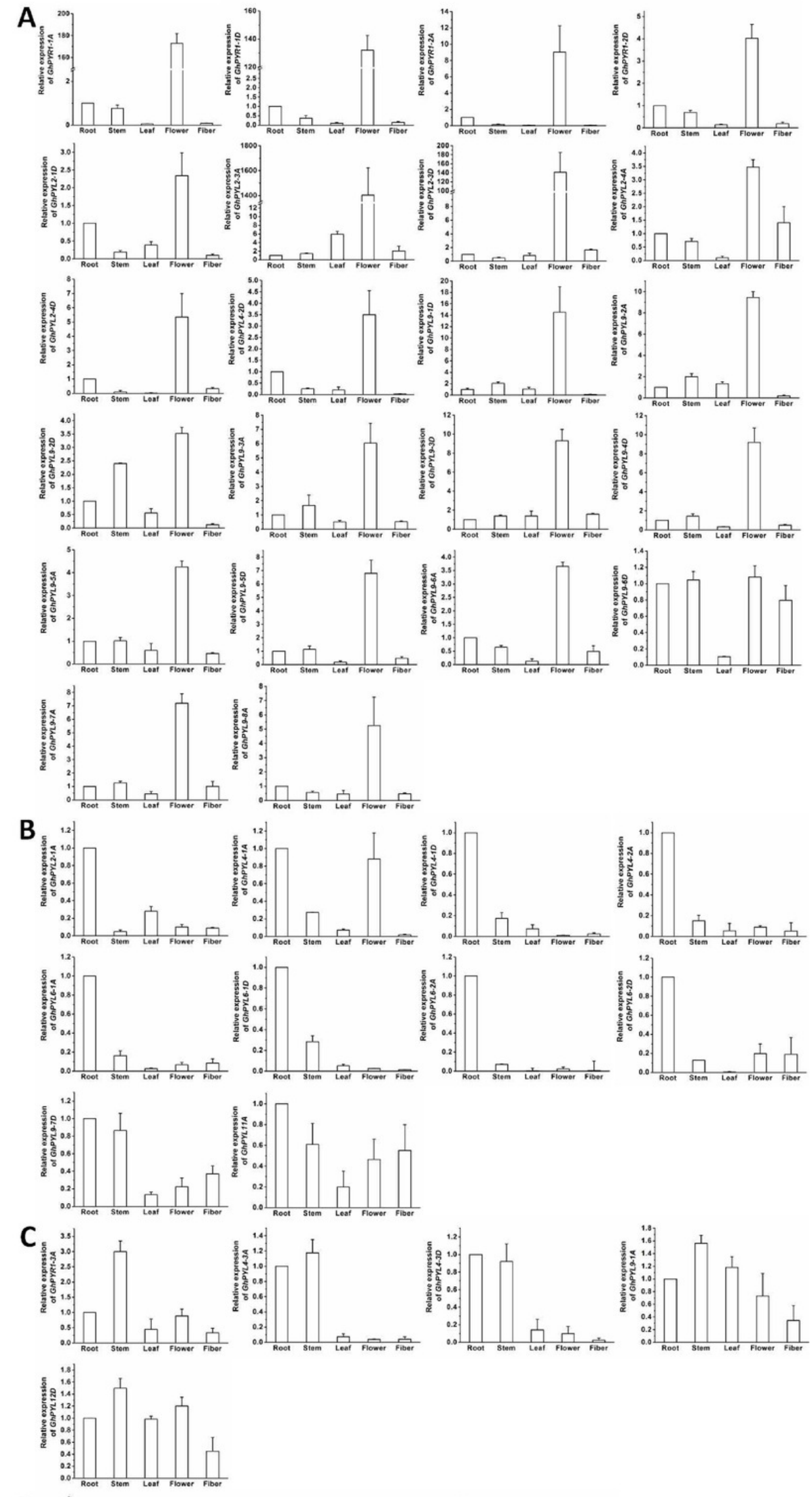

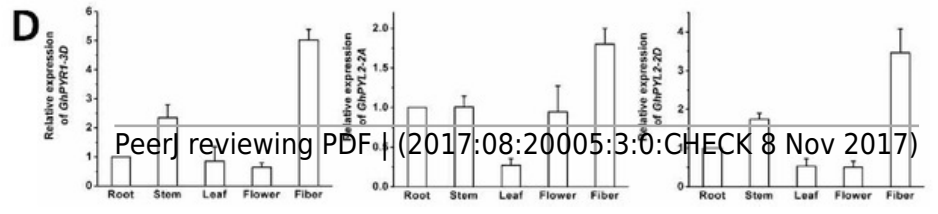




\section{Figure 8}

Expression of GhPYL genes in response to ABA. The relative expression of GhPYLs was examined after treatments with $100 \mu \mathrm{M}$ ABA for indicated period of time.

The expression levels of the genes were markedly decreased at $3 \mathrm{~h}$ or $6 \mathrm{~h}$ but increased at $12 \mathrm{~h}$ or $24 \mathrm{~h}(\mathrm{~A})$, were continually increased (B), decreased (C), and increased at $3 \mathrm{~h}$ or $6 \mathrm{~h}$ but decreased at $12 \mathrm{~h}$ or $24 \mathrm{~h}(\mathrm{D})$, and were not altered (E). Cotton gene GhUBQ7 was applied as the internal control. The gene expression value at $0 \mathrm{~h}$ was set as 1 . The values are mean $\pm \mathrm{SE}$. 
PeerJ

Manuscript to be reviewed

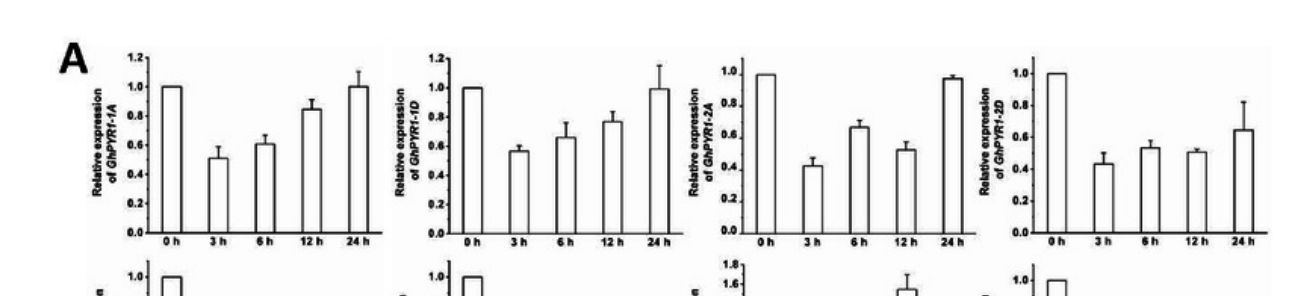

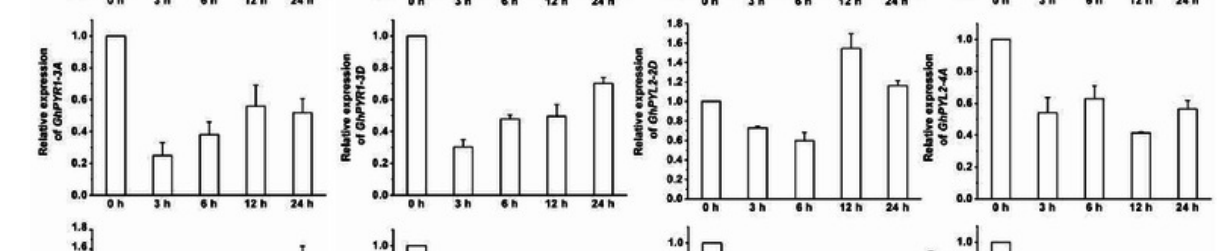

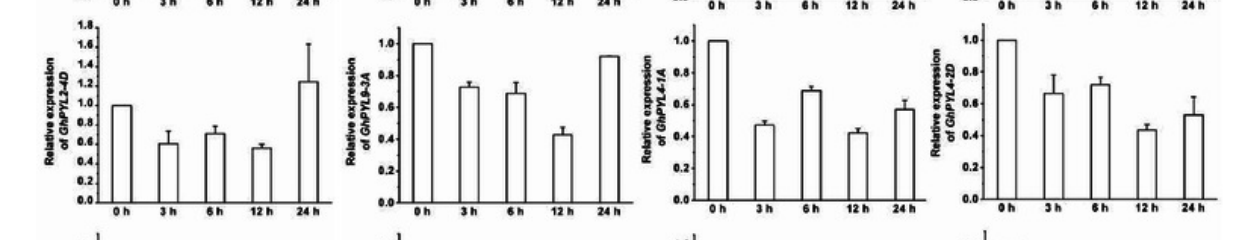

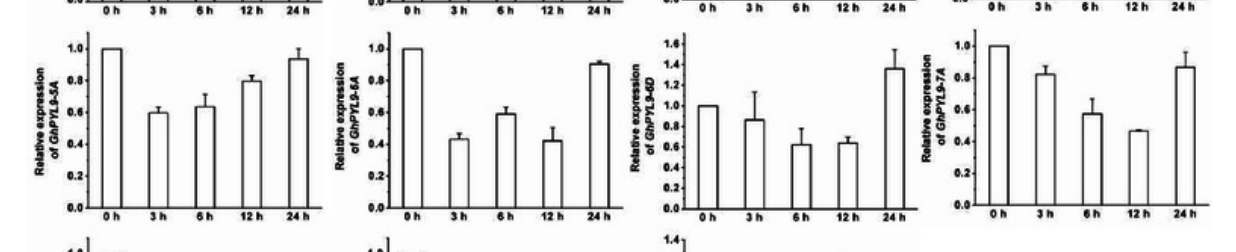

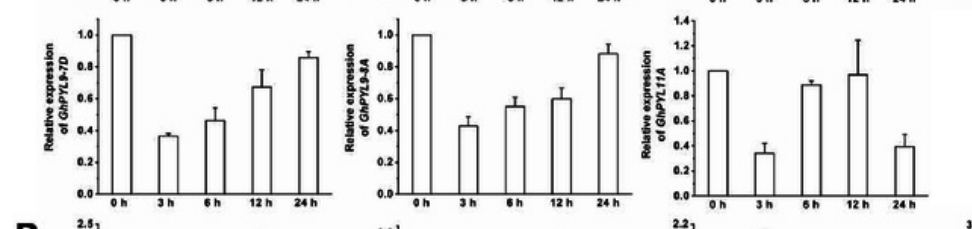

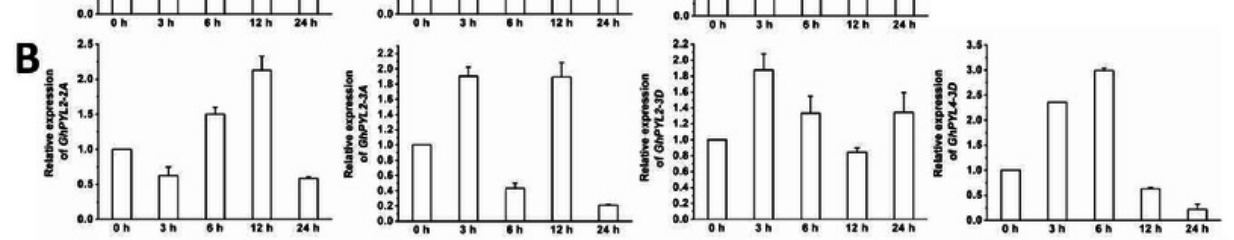

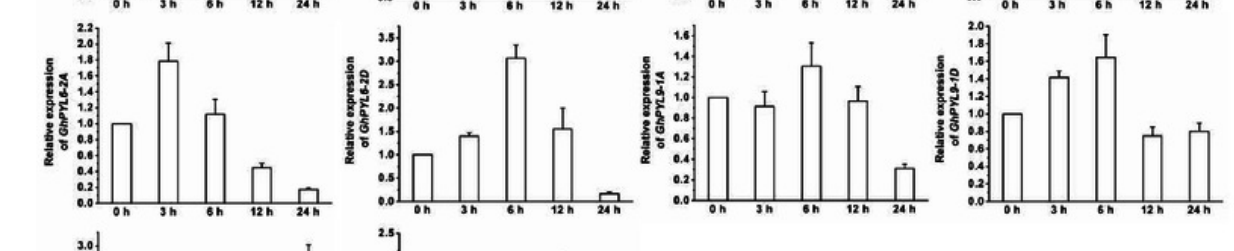

$$
\begin{aligned}
& \text { Homoth }
\end{aligned}
$$

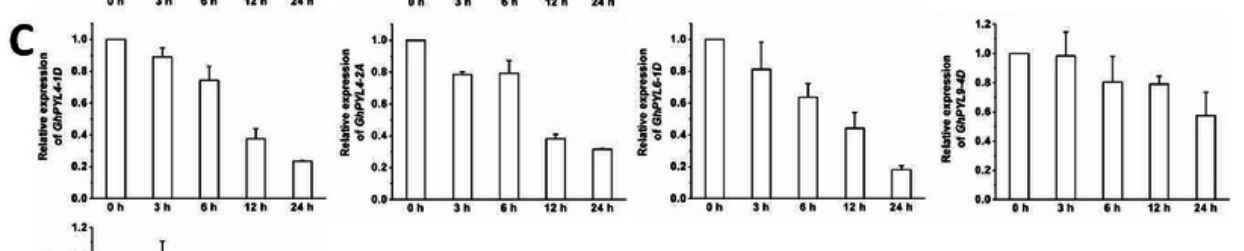

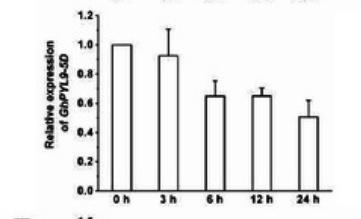

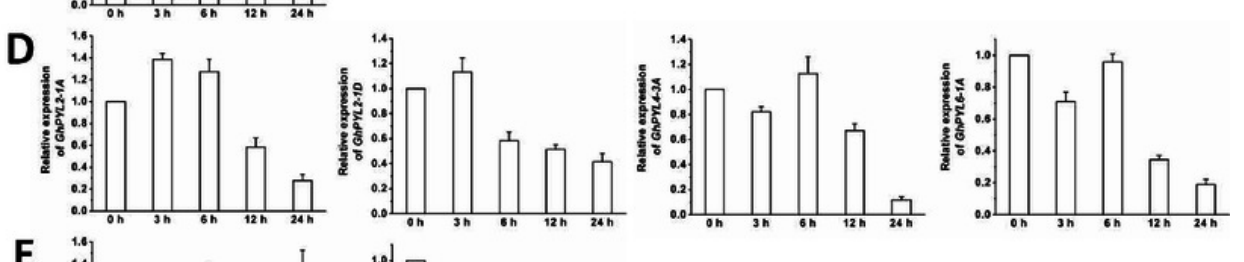




\section{Figure 9}

Expression of GhPYL genes in response to osmotic stress.

The relative expression of GhPYLs was analyzed after treatments with 10\% PEG6000 for indicated period of time. The transcriptional levels of the genes were remarkably increased (A), decreased (B), and not altered (C). Gene GhUBQ7 was applied as the internal control. The gene expression value at $0 \mathrm{~h}$ was set as 1 . The values are mean $\pm \mathrm{SE}$. 
A
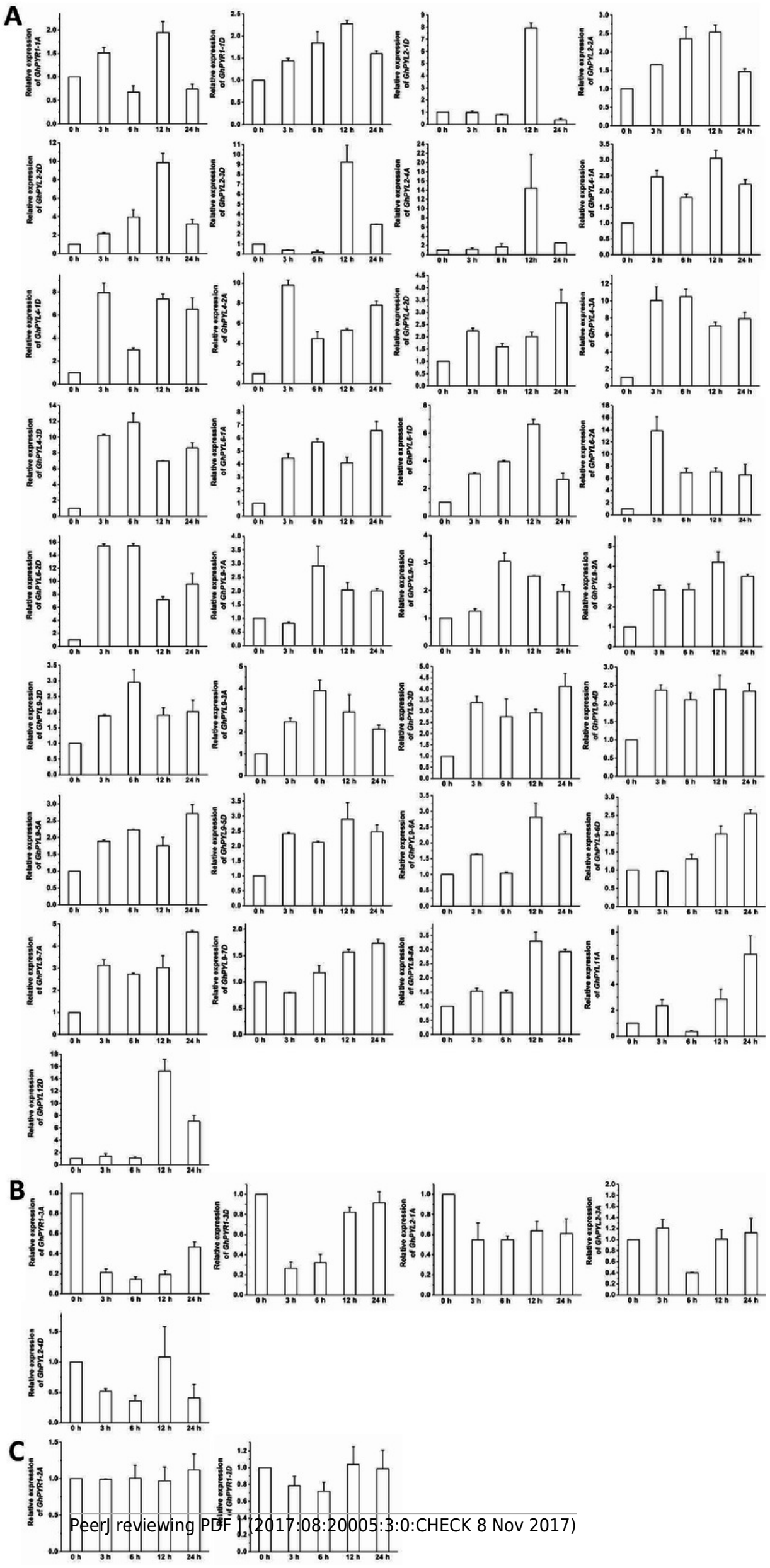


\section{Figure 10}

\section{Analysis of interactions between GhPYLs and GhABIIA or GhABIID.}

Interactions between GhPYLs and GhABIIA (A), and between GhPYLs and GhABI1D (B) were examined by yeast two-hybrid method. The reduced cell densities in the dilution series are revealed by narrowing triangles when proceeding from left to right. 


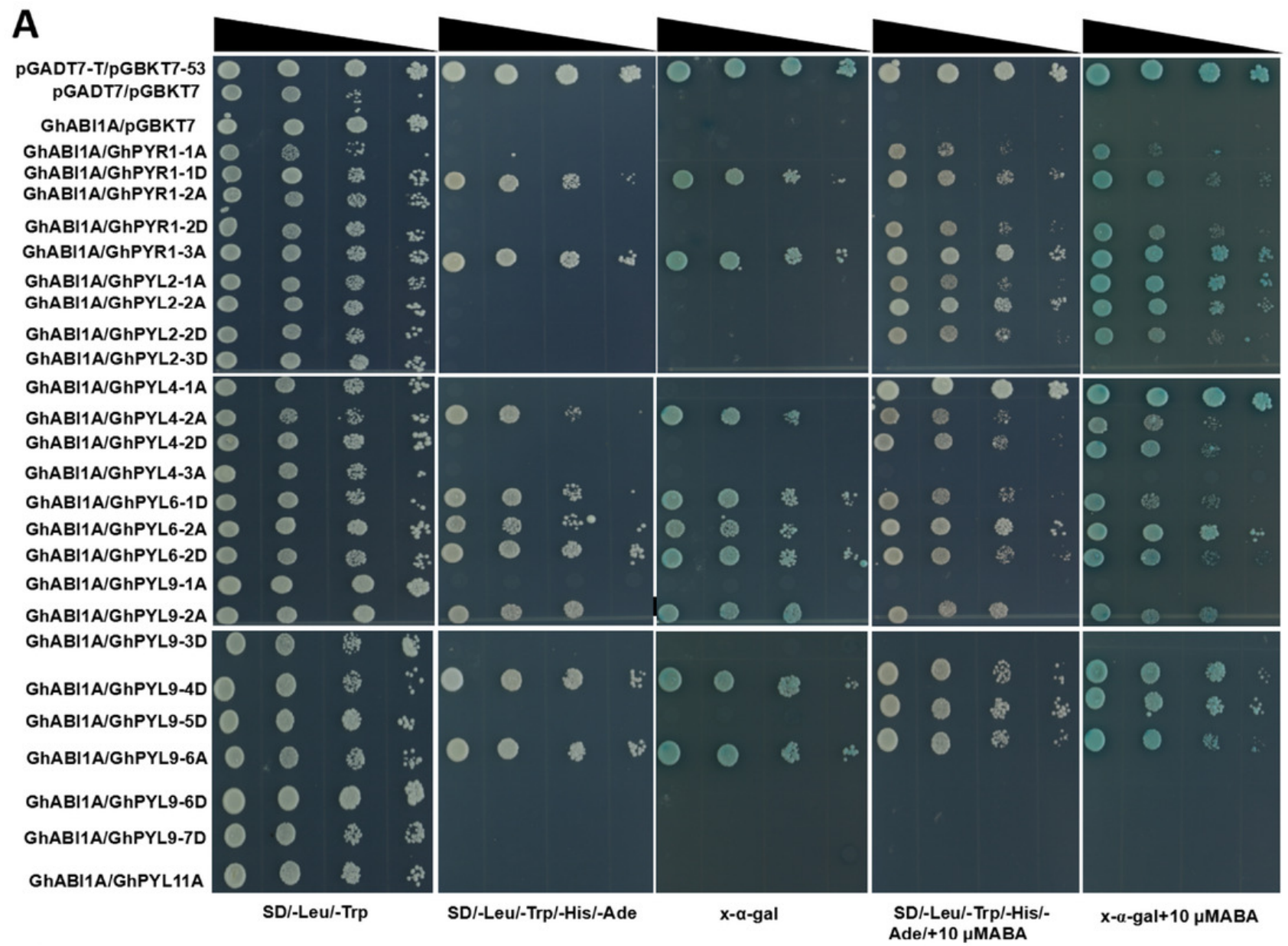

B

pGADT7-T/PGBKT7-53 pGADT7/pGBKT7 GhABI1D/pGBKT7 GhABI1D/GhPYR1-1A GhABI1D/GhPYR1-1D GhABI1D/GhPYR1-2A GhABI1D/GhPYR1-2D GhABI1D/GhPYR1-3A GhABI1D/GhPYL2-1A GhABI1D/GhPYL2-2A GhABI1D/GhPYL2-2D GhABI1D/GhPYL2-3D GhABI1D/GhPYL4-1A GhABI1D/GhPYL4-2A GhABI1D/GhPYL4-2D GhABI1D/GhPYL4-3A GhABI1D/GhPYL6-1D GhABI1D/GhPYL6-2A GhABI1D/GhPYL6-2D GhABI1D/GhPYL9-1A GhABI1D/GhPYL9-2A GhABI1D/GhPYL9-3D GhABI1D/GhPYL9-4D GhABI1D/GhPYL9-5D GhABI1D/GhPYL9-6A GhABI1D/GhPYL9-6D GhABI1D/GhPYL9-7D

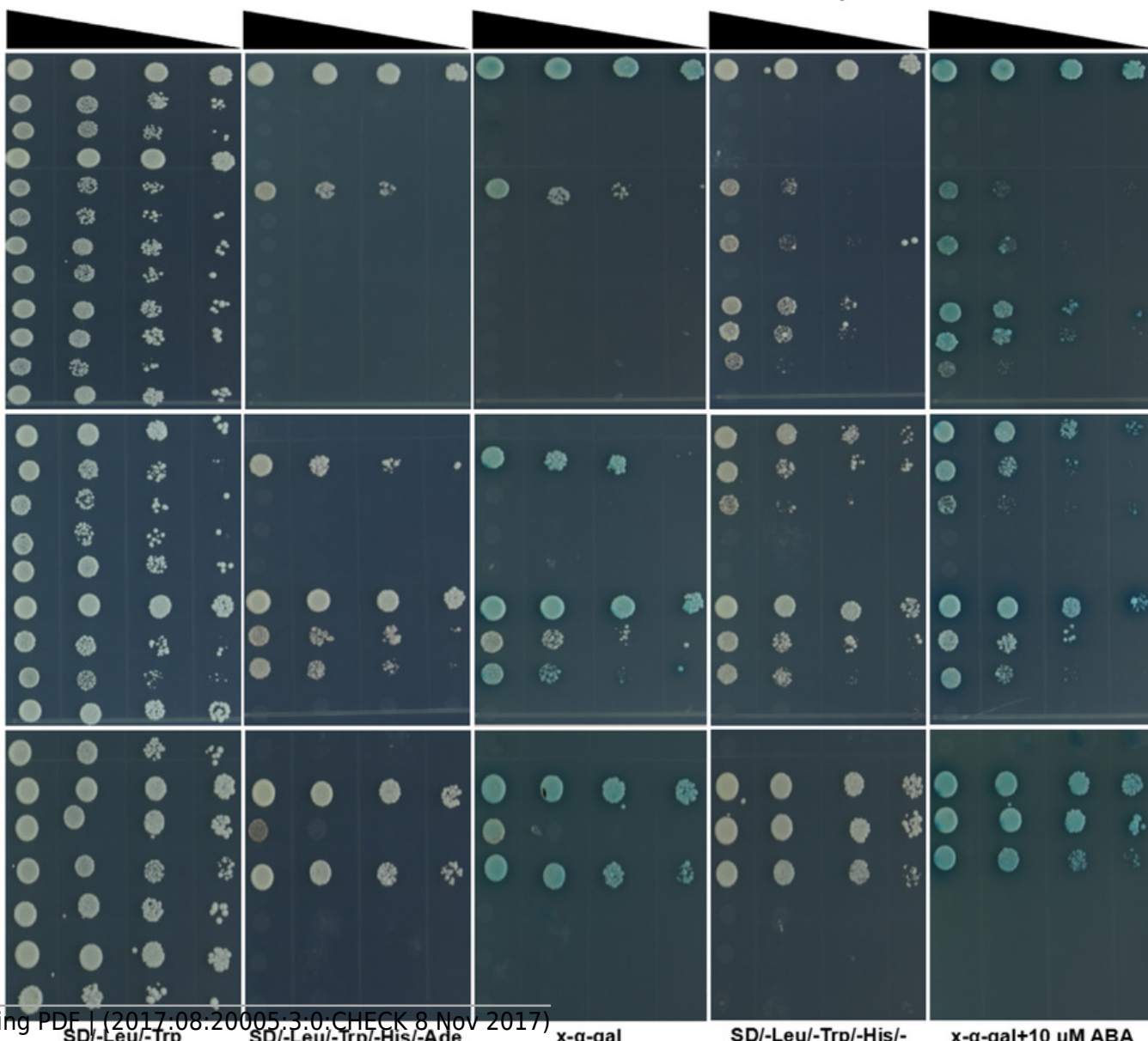




\section{Table 1 (on next page)}

Gene nomenclature and homolog table. 
Table 1 Gene nomenclature and homolog table

\begin{tabular}{|c|c|c|c|c|c|c|c|c|c|c|}
\hline Homologs & G. arboretum & G. raimondii & G. hirsutum & G. barbadense & A. thaliana & T. cacao & R. communis & V. vinifera & B. distachyon & O. sativa \\
\hline 1 & $\begin{array}{l}\text { Cotton_A_13 } \\
999 \\
\text { (GaPYL2-1) }\end{array}$ & $\begin{array}{l}\text { 009G045700 } \\
\text { (GrPYL2-1) }\end{array}$ & $\begin{array}{l}\text { Gh_A05G0336 } \\
\text { (GhPYL2-1A)/ } \\
\text { Gh_D05G0441 } \\
\text { (GhPYL2-1D) } \\
\end{array}$ & $\begin{array}{l}\text { AA31030 } \\
\text { (GbPYL2-1A)/ } \\
\text { DD16128 } \\
\text { (GbPYL2-1D) } \\
\end{array}$ & $\begin{array}{l}\text { AT2G26040 } \\
\text { (AtPYL2) }\end{array}$ & & $\begin{array}{l}\text { 29794.m0033 } \\
\text { 35(RcPYRL2) }\end{array}$ & $\begin{array}{l}\text { GSVIVT01 } \\
\text { 035362001 } \\
\text { (VvPYRL2) }\end{array}$ & $\begin{array}{l}\text { BRADI1G37810(BdPYRL2)/ } \\
\text { BRADI3G08580(BdPYRL3) }\end{array}$ & $\begin{array}{l}\text { Os06t0562200 } \\
\text { (OsPYL2)/ } \\
\text { Os02t0226801 } \\
\text { (OsPYL3) } \\
\end{array}$ \\
\hline 2 & $\begin{array}{l}\text { Cotton_A_11 } \\
886 \\
\text { (GaPYL2-2) }\end{array}$ & $\begin{array}{l}\text { 004G287300 } \\
\text { (GrPYL2-2) }\end{array}$ & $\begin{array}{l}\text { Gh_A08G2221 } \\
\text { (GhPYL2-2A)/ } \\
\text { Gh_D08G2587 } \\
\text { (GhPYL2-2D) }\end{array}$ & $\begin{array}{l}\text { AA34547 } \\
\text { (GbPYL2-2A)/ } \\
\text { DD10391 } \\
\text { (GbPYL2-2D)/ } \\
\text { DD38339 } \\
\text { (GbPYL2-2D') }\end{array}$ & $\begin{array}{l}\text { AT2G26040 } \\
\text { (AtPYL2) }\end{array}$ & & & & $\begin{array}{l}\text { BRADI1G37810(BdPYRL2)/ } \\
\text { BRADI3G08580(BdPYRL3) }\end{array}$ & $\begin{array}{l}\text { Os06t0562200 } \\
\text { (OsPYL2)/ } \\
\text { Os02t0226801 } \\
\text { (OsPYL3) }\end{array}$ \\
\hline 3 & $\begin{array}{l}\text { Cotton_A_05 } \\
614 \\
\text { (GaPYL2-3) }\end{array}$ & $\begin{array}{l}\text { 001G023000 } \\
\text { (GrPYL2-3) }\end{array}$ & $\begin{array}{l}\text { Gh_A07G2326 } \\
\text { (GhPYL2-3A)/ } \\
\text { Gh_D07G0193 } \\
\text { (GhPYL2-3D) }\end{array}$ & $\begin{array}{l}\text { AA28868 } \\
\text { (GbPYL2-3A)/ } \\
\text { DD32447 } \\
\text { (GbPYL2-3D) } \\
\end{array}$ & $\begin{array}{l}\text { AT2G26040 } \\
\text { (AtPYL2) }\end{array}$ & & & & $\begin{array}{l}\text { BRADI1G37810(BdPYRL2)/ } \\
\text { BRADI3G08580(BdPYRL3) }\end{array}$ & $\begin{array}{l}\text { Os06t0562200 } \\
\text { (OsPYL2)/ } \\
\text { Os02t0226801 } \\
\text { (OsPYL3) }\end{array}$ \\
\hline 4 & $\begin{array}{l}\text { Cotton_A_11 } \\
801 \\
\text { (GaPYL2-4) }\end{array}$ & $\begin{array}{l}\text { 011G081100 } \\
\text { (GrPYL2-4) }\end{array}$ & $\begin{array}{l}\text { Gh_A10G0677 } \\
\text { (GhPYL2-4A)/ } \\
\text { Gh_D10G0710 } \\
\text { (GhPYL2-4D) } \\
\end{array}$ & $\begin{array}{l}\text { AA22659 } \\
\text { (GbPYL2-4A)/ } \\
\text { DD23620 } \\
\text { (GbPYL2-4D) } \\
\end{array}$ & $\begin{array}{l}\text { AT2G26040 } \\
\text { (AtPYL2)/ } \\
\text { AT1G73000 } \\
\text { (AtPYL3) } \\
\end{array}$ & $\begin{array}{l}\text { Thecc1EG0 } \\
29025 \\
\text { (TcPYRL3) }\end{array}$ & & & $\begin{array}{l}\text { BRADI1G37810(BdPYRL2)/ } \\
\text { BRADI3G08580(BdPYRL3) }\end{array}$ & $\begin{array}{l}\text { Os06t0562200 } \\
\text { (OsPYL2)/ } \\
\text { Os02t0226801 } \\
\text { (OsPYL3) }\end{array}$ \\
\hline 5 & $\begin{array}{l}\text { Cotton_A_00 } \\
296 \\
\text { (GaPYL4-1) }\end{array}$ & $\begin{array}{l}\text { 002G266100 } \\
\text { (GrPYL4-2) }\end{array}$ & $\begin{array}{l}\text { Gh_A01G1990 } \\
\text { (GhPYL4-1A)/ } \\
\text { Gh_D01G2250 } \\
\text { (GhPYL4-2D) }\end{array}$ & $\begin{array}{l}\text { AA24072 } \\
\text { (GbPYL4-1A) }\end{array}$ & $\begin{array}{l}\text { AT2G38310 } \\
\text { (AtPYL4)/ } \\
\text { AT5G05440 } \\
\text { (AtPYL5)/ } \\
\text { AT2G40330 } \\
\text { (AtPYL6) }\end{array}$ & $\begin{array}{l}\text { Thecc1EG0 } \\
21605 \\
\text { (TcPYRL4) }\end{array}$ & $\begin{array}{l}\text { 29820.m0010 } \\
\text { 02(RcPYRL3) }\end{array}$ & & $\begin{array}{l}\text { BRADI2G22510(BdPYRL4)/ } \\
\text { BRADI1G16710(BdPYRL5)/ } \\
\text { BRADI1G65130(BdPYRL6)/ } \\
\text { BRADI2G53840(BdPYLR7) }\end{array}$ & $\begin{array}{l}\text { Os01t0827800 } \\
\text { (OsPYL4)/ } \\
\text { Os05t0473000 } \\
\text { (OsPYL5)/ } \\
\text { Os03t0297600 } \\
\text { (OsPYL6) }\end{array}$ \\
\hline 6 & $\begin{array}{l}\text { Cotton_A_22 } \\
319 \\
\text { (GaPYL4-2) }\end{array}$ & $\begin{array}{l}\text { 006G185000 } \\
\text { (GrPYL4-1) }\end{array}$ & $\begin{array}{l}\text { Gh_A09G2421 } \\
\text { (GhPYL4-2A)/ } \\
\text { Gh_D09G1585 } \\
\text { (GhPYL4-1D) }\end{array}$ & $\begin{array}{l}\text { AA31755 } \\
\text { (GbPYL4-2A)/ } \\
\text { DD31762 } \\
\text { (GbPYL4-1D) }\end{array}$ & $\begin{array}{l}\text { AT2G38310 } \\
\text { (AtPYL4)/ } \\
\text { AT5G05440 } \\
\text { (AtPYL5)/ } \\
\text { AT2G40330 } \\
\text { (AtPYL6) }\end{array}$ & $\begin{array}{l}\text { Thecc1EG0 } \\
21605 \\
\text { (TcPYRL4) }\end{array}$ & $\begin{array}{l}\text { 29820.m0010 } \\
\text { 02(RcPYRL3) }\end{array}$ & & $\begin{array}{l}\text { BRADI2G22510(BdPYRL4)/ } \\
\text { BRADI1G16710(BdPYRL5)/ } \\
\text { BRADI1G65130(BdPYRL6)/ } \\
\text { BRADI2G53840(BdPYLR7) }\end{array}$ & $\begin{array}{l}\text { Os01t0827800 } \\
\text { (OsPYL4)/ } \\
\text { Os05t0473000 } \\
\text { (OsPYL5)/ } \\
\text { Os03t0297600 } \\
\text { (OsPYL6) }\end{array}$ \\
\hline 7 & $\begin{array}{l}\text { Cotton_A_31 } \\
479 \\
\text { (GaPYL4-3) }\end{array}$ & $\begin{array}{l}\text { 009G323000 } \\
\text { (GrPYL4-3) }\end{array}$ & $\begin{array}{l}\text { Gh_A05G2630 } \\
\text { (GhPYL4-3A)/ } \\
\text { Gh_D05G2920 } \\
\text { (GhPYL4-3D) }\end{array}$ & $\begin{array}{l}\text { AA01168 } \\
\text { (GbPYL4-3A)/ } \\
\text { DD09701 } \\
\text { (GbPYL4-3D) }\end{array}$ & $\begin{array}{l}\text { AT2G38310 } \\
\text { (AtPYL4)/ } \\
\text { AT5G05440 } \\
\text { (AtPYL5)/ } \\
\text { AT2G40330 } \\
\text { (AtPYL6) }\end{array}$ & $\begin{array}{l}\text { Thecc1EG0 } \\
42432 \\
\text { (TcPYRL5) }\end{array}$ & & & $\begin{array}{l}\text { BRADI2G22510(BdPYRL4)/ } \\
\text { BRADI1G16710(BdPYRL5)/ } \\
\text { BRADI1G65130(BdPYRL6)/ } \\
\text { BRADI2G53840(BdPYLR7) }\end{array}$ & $\begin{array}{l}\text { Os01t0827800 } \\
\text { (OsPYL4)/ } \\
\text { Os05t0473000 } \\
\text { (OsPYL5)/ } \\
\text { Os03t0297600 } \\
\text { (OsPYL6) }\end{array}$ \\
\hline 8 & $\begin{array}{l}\text { Cotton_A_15 } \\
835 \\
\text { (GaPYL6-1) }\end{array}$ & $\begin{array}{l}\text { 011G290300 } \\
\text { (GrPYL6-1) }\end{array}$ & $\begin{array}{l}\text { Gh_A10G2142 } \\
\text { (GhPYL6-1A)/ } \\
\text { Gh_D10G2388 } \\
\text { (GhPYL6-1D) }\end{array}$ & $\begin{array}{l}\text { AA18332 } \\
\text { (GbPYL6-1A)/ } \\
\text { DD29255 } \\
\text { (GbPYL6-1D) }\end{array}$ & $\begin{array}{l}\text { AT2G38310 } \\
\text { (AtPYL4)/ } \\
\text { AT5G05440 } \\
\text { (AtPYL5)/ } \\
\text { AT2G40330 } \\
\text { (AtPYL6) }\end{array}$ & & & & $\begin{array}{l}\text { BRADI2G22510(BdPYRL4)/ } \\
\text { BRADI1G16710(BdPYRL5)/ } \\
\text { BRADI1G65130(BdPYRL6)/ } \\
\text { BRADI2G53840(BdPYLR7) }\end{array}$ & $\begin{array}{l}\text { Os01t0827800 } \\
\text { (OsPYL4)/ } \\
\text { Os05t0473000 } \\
\text { (OsPYL5)/ } \\
\text { Os03t0297600 } \\
\text { (OsPYL6) }\end{array}$ \\
\hline 9 & $\begin{array}{l}\text { Cotton_A_17 } \\
533\end{array}$ & & & $\begin{array}{l}\text { AA15331 } \\
\text { (GbPYL6-2A')/ }\end{array}$ & $\begin{array}{l}\text { AT5G45860 } \\
\text { (AtPYL11)/ }\end{array}$ & $\begin{array}{l}\text { Thecc1EG0 } \\
29689\end{array}$ & & & & \\
\hline
\end{tabular}




\begin{tabular}{|c|c|c|c|c|c|c|c|c|c|c|}
\hline & (GaPYL6-2) & & & $\begin{array}{l}\text { DD25577 } \\
\text { (GbPYL6-2D') }\end{array}$ & $\begin{array}{l}\text { AT5G45870 } \\
\text { (AtPYL12)/ } \\
\text { AT4G18620 } \\
\text { (AtPYL13) }\end{array}$ & (TcPYL6) & & & & \\
\hline 10 & $\begin{array}{l}\text { Cotton_A_24 } \\
449 \\
\text { (GaPYL9-1) }\end{array}$ & $\begin{array}{l}\text { 004G152300 } \\
\text { (GrPYL9-2) }\end{array}$ & $\begin{array}{l}\text { Gh_A08G1117 } \\
\text { (GhPYL9-1A)/ } \\
\text { Gh_D08G1399 } \\
\text { (GhPYL9-2D) }\end{array}$ & $\begin{array}{l}\text { DD28661 } \\
\text { (GbPYL9-2D) }\end{array}$ & $\begin{array}{l}\text { AT4G01026 } \\
\text { (AtPYL7)/ } \\
\text { AT1G01360 } \\
\text { (AtPYL9)/ } \\
\text { AT4G27920 } \\
\text { (AtPYL10) }\end{array}$ & $\begin{array}{l}\text { Thecc1EG0 } \\
05169 \\
\text { (TcPYRL9) }\end{array}$ & & $\begin{array}{l}\text { GSVIVT01 } \\
\text { 027078001 } \\
\text { (VvPYRL5) }\end{array}$ & BRADI2G32250(BdPYRL8) & $\begin{array}{l}\text { Os05t0213500 } \\
\text { (OsPYRL11) }\end{array}$ \\
\hline 11 & $\begin{array}{l}\text { Cotton_A_14 } \\
394 \\
\text { (GaPYL9-2) }\end{array}$ & $\begin{array}{l}\text { 007G107500 } \\
\text { (GrPYL9-1) }\end{array}$ & $\begin{array}{l}\text { Gh_A11G0870 } \\
\text { (GhPYL9-2A)/ } \\
\text { Gh_D11G1013 } \\
\text { (GhPYL9-1D) }\end{array}$ & $\begin{array}{l}\text { DD37081 } \\
\text { (GbPYL9-1D) }\end{array}$ & $\begin{array}{l}\text { AT4G01026 } \\
\text { (AtPYL7)/ } \\
\text { AT1G01360 } \\
\text { (AtPYL9)/ } \\
\text { AT4G27920 } \\
\text { (AtPYL10) }\end{array}$ & $\begin{array}{l}\text { Thecc1EG0 } \\
\text { 05169 } \\
\text { (TcPYRL9) }\end{array}$ & & $\begin{array}{l}\text { GSVIVT01 } \\
\text { 027078001 } \\
\text { (VvPYRL5) }\end{array}$ & $\begin{array}{l}\text { BRADI2G32250(BdPYRL8)/ } \\
\text { BRADI3G09580(BdPYRL9) }\end{array}$ & $\begin{array}{l}\text { Os02t0255500 } \\
\text { (OsPYL10)/ } \\
\text { Os05t0213500 } \\
\text { (OsPYL11) }\end{array}$ \\
\hline 12 & $\begin{array}{l}\text { Cotton_A_36 } \\
156 \\
\text { (GaPYL9-3) }\end{array}$ & $\begin{array}{l}\text { 007G026100 } \\
\text { (GrPYL9-4) }\end{array}$ & $\begin{array}{l}\text { Gh_A11G0224 } \\
\text { (GhPYL9-3A)/ } \\
\text { Gh_D11G0238 } \\
\text { (GhPYL9-4D) }\end{array}$ & $\begin{array}{l}\text { AA40052 } \\
(\text { GbPYL9-3A)/ } \\
\text { DD15345 } \\
\text { (GbPYL9-4D) }\end{array}$ & $\begin{array}{l}\text { AT4G01026 } \\
\text { (AtPYL7)/ } \\
\text { AT1G01360 } \\
\text { (AtPYL9)/ } \\
\text { AT4G27920 } \\
\text { (AtPYL10) } \\
\end{array}$ & & $\begin{array}{l}\text { 29742.m0014 } \\
\text { 42(RcPYRL5) }\end{array}$ & $\begin{array}{l}\text { GSVIVT01 } \\
019517001 \\
\text { (VvPYRL3) }\end{array}$ & & \\
\hline 13 & $\begin{array}{l}\text { Cotton_A_13 } \\
258 \\
\text { (GaPYL9-4)/ } \\
\text { Cotton_A_23 } \\
205 \\
\text { (GaPYL9-5) }\end{array}$ & $\begin{array}{l}\text { 008G253700 } \\
\text { (GrPYL9-5) }\end{array}$ & $\begin{array}{l}\text { Gh_A12G2127 } \\
\text { (GhPYL9-5A)/ } \\
\text { Gh_D12G2306 } \\
\text { (GhPYL9-5D) }\end{array}$ & $\begin{array}{l}\text { AA38557 } \\
(\text { GbPYL9-5A)/ } \\
\text { DD26619 } \\
\text { (GbPYL9-5D) }\end{array}$ & $\begin{array}{l}\text { AT4G01026 } \\
\text { (AtPYL7)/ } \\
\text { AT1G01360 } \\
\text { (AtPYL9)/ } \\
\text { AT4G27920 } \\
\text { (AtPYL10) }\end{array}$ & $\begin{array}{l}\text { Thecc1EG0 } \\
16450 \\
\text { (TcPYRL7) }\end{array}$ & $\begin{array}{l}\text { 29742.m0014 } \\
\text { 42(RcPYRL5) }\end{array}$ & $\begin{array}{l}\text { GSVIVT01 } \\
019517001 \\
\text { (VvPYRL3) }\end{array}$ & BRADI3G09580(BdPYRL9) & $\begin{array}{l}\text { Os02t0255500 } \\
\text { (OsPYL10) }\end{array}$ \\
\hline 14 & $\begin{array}{l}\text { Cotton_A_08 } \\
084 \\
\text { (GaPYL9-6) }\end{array}$ & $\begin{array}{l}\text { 006G200700 } \\
\text { (GrPYL9-6) }\end{array}$ & $\begin{array}{l}\text { Gh_A09G1646 } \\
\text { (GhPYL9-6A)/ } \\
\text { Gh_D09G1740 } \\
\text { (GhPYL9-6D) }\end{array}$ & $\begin{array}{l}\text { AA22571 } \\
\text { (GbPYL9-6A)/ } \\
\text { DD17050 } \\
\text { (GbPYL9-6D)/ } \\
\text { DD32170 } \\
\text { (GbPYL9-6D') }\end{array}$ & $\begin{array}{l}\text { AT4G01026 } \\
\text { (AtPYL7)/ } \\
\text { AT1G01360 } \\
\text { (AtPYL9)/ } \\
\text { AT4G27920 } \\
\text { (AtPYL10) } \\
\end{array}$ & & $\begin{array}{l}\text { 30169.m0065 } \\
\text { 25(RcPYRL6) }\end{array}$ & & BRADI3G09580(BdPYRL9) & $\begin{array}{l}\text { Os02t0255500 } \\
\text { (OsPYL10) }\end{array}$ \\
\hline 15 & $\begin{array}{l}\text { Cotton_A_20 } \\
366 \\
\text { (GaPYL9-7) }\end{array}$ & $\begin{array}{l}\text { 012G003800 } \\
\text { (GrPYL9-3) }\end{array}$ & $\begin{array}{l}\text { Gh_A05G3585 } \\
\text { (GhPYL9-7A)/ } \\
\text { Gh_D04G0019 } \\
\text { (GhPYL9-3D) }\end{array}$ & $\begin{array}{l}\text { DD31179 } \\
\text { (GbPYL9-3D) }\end{array}$ & $\begin{array}{l}\text { AT4G01026 } \\
\text { (AtPYL7)/ } \\
\text { AT1G01360 } \\
\text { (AtPYL9)/ } \\
\text { AT4G27920 } \\
\text { (AtPYL10) }\end{array}$ & & $\begin{array}{l}\text { 30169.m0065 } \\
\text { 25(RcPYRL6) }\end{array}$ & & BRADI3G09580(BdPYRL9) & $\begin{array}{l}\text { Os02t0255500 } \\
\text { (OsPYL10) }\end{array}$ \\
\hline 16 & $\begin{array}{l}\text { Cotton_A_17 } \\
740 \\
\text { (GaPYL9-8) }\end{array}$ & $\begin{array}{l}\text { 008G270200 } \\
\text { (GrPYL9-7) }\end{array}$ & $\begin{array}{l}\text { Gh_A12G2278 } \\
\text { (GhPYL9-8A)/ } \\
\text { Gh_D12G2694 } \\
\text { (GhPYL9-7D) }\end{array}$ & $\begin{array}{l}\text { DD31997 } \\
\text { (GbPYL9-7D) }\end{array}$ & $\begin{array}{l}\text { AT4G01026 } \\
\text { (AtPYL7)/ } \\
\text { AT1G01360 } \\
\text { (AtPYL9)/ } \\
\text { AT4G27920 } \\
\text { (AtPYL10) }\end{array}$ & & $\begin{array}{l}\text { 30190.m0108 } \\
\text { 24(RcPYRL7) }\end{array}$ & & BRADI3G09580(BdPYRL9) & $\begin{array}{l}\text { Os02t0255500 } \\
\text { (OsPYL10) }\end{array}$ \\
\hline 17 & Cotton_A 11 & 009G162000 & Gh A05G1297( & AA37880(GbP & AT5G45860 & & & & & \\
\hline
\end{tabular}




\begin{tabular}{|c|c|c|c|c|c|c|c|c|c|c|}
\hline & $\begin{array}{l}270 \\
\text { (GaPYL11) }\end{array}$ & (GrPYL12) & $\begin{array}{l}\text { GhPYL11A)/ } \\
\text { Gh_D05G1468( } \\
\text { GhPYL12D) }\end{array}$ & $\begin{array}{l}\text { YL11A)/ } \\
\text { DD17498(GbP } \\
\text { YL12D) }\end{array}$ & $\begin{array}{l}\text { (AtPYL11)/ } \\
\text { AT5G45870 } \\
\text { (AtPYL12)/ } \\
\text { AT4G18620 } \\
\text { (AtPYL13) }\end{array}$ & & & & & \\
\hline 18 & $\begin{array}{l}\text { Cotton_A_23 } \\
118 \\
\text { (GaPYR1-1) }\end{array}$ & $\begin{array}{l}\text { 003G181600 } \\
\text { (GrPYR1-1) }\end{array}$ & $\begin{array}{l}\text { Gh_A03G0015 } \\
\text { (GhPYR1-1A)/ } \\
\text { Gh_D03G1860 } \\
\text { (GhPYR1-1D) }\end{array}$ & $\begin{array}{l}\text { DD23506 } \\
\text { (GbPYR1-1D) }\end{array}$ & $\begin{array}{l}\text { AT4G17870 } \\
\text { (AtPYR1)/ } \\
\text { AT5G46790 } \\
\text { (AtPYL1) }\end{array}$ & & & $\begin{array}{l}\text { GSVIVT01 } \\
013161001 \\
\text { (VvPYRL1) }\end{array}$ & BRADI3G34070(BdPYRL1) & $\begin{array}{l}\text { Os02t0255500 } \\
\text { (OsPYL1) }\end{array}$ \\
\hline 19 & $\begin{array}{l}\text { Cotton_A_09 } \\
168 \\
\text { (GaPYR1-2) }\end{array}$ & $\begin{array}{l}\text { 007G031800 } \\
\text { (GrPYR1-2) }\end{array}$ & $\begin{array}{l}\text { Gh_A11G0270 } \\
\text { (GhPYR1-2A)/ } \\
\text { Gh_D11G0290 } \\
\text { (GhPYR1-2D) }\end{array}$ & $\begin{array}{l}\text { DD21981 } \\
\text { (GbPYR1-2D) }\end{array}$ & $\begin{array}{l}\text { AT4G17870 } \\
\text { (AtPYR1)/ } \\
\text { AT5G46790 } \\
\text { (AtPYL1) }\end{array}$ & $\begin{array}{l}\text { Thecc1EG0 } \\
15719 \\
\text { (TcPYRL1) }\end{array}$ & $\begin{array}{l}\text { 29827.m0025 } \\
\text { 33(RcPYRL1) }\end{array}$ & $\begin{array}{l}\text { GSVIVT01 } \\
013161001 \\
\text { (VvPYRL1) }\end{array}$ & BRADI3G34070(BdPYRL1) & $\begin{array}{l}\text { Os02t0255500 } \\
\text { (OsPYL1) }\end{array}$ \\
\hline 20 & $\begin{array}{l}\text { Cotton_A_07 } \\
\text { 906(GaPYR1- } \\
\text { 3) }\end{array}$ & $\begin{array}{l}\text { 008G226500( } \\
\text { GrPYR1-3) }\end{array}$ & $\begin{array}{l}\text { Gh_A12G1895 } \\
\text { (GhPYR1-3A)/ } \\
\text { Gh_D12G2076 } \\
\text { (GhPYR1-3D) }\end{array}$ & $\begin{array}{l}\text { AA29004 } \\
(\text { GbPYR1-3A)/ } \\
\text { DD35789 } \\
\text { (GbPYR1-3D) }\end{array}$ & $\begin{array}{l}\text { AT4G17870 } \\
\text { (AtPYR1)/ } \\
\text { AT5G46790 } \\
\text { (AtPYL1) }\end{array}$ & & & & BRADI3G34070(BdPYRL1) & $\begin{array}{l}\text { Os02t0255500 } \\
\text { (OsPYL1) }\end{array}$ \\
\hline 21 & & $\begin{array}{l}\text { 010G194800 } \\
\text { (GrPYL6-2) }\end{array}$ & $\begin{array}{l}\text { Gh_A06G1418 } \\
\text { (GhPYL6-2A)/ } \\
\text { Gh_D06G1764( } \\
\text { GhPYL6-2D) }\end{array}$ & $\begin{array}{l}\text { AA13391 } \\
\text { (GbPYL6-2A)/ } \\
\text { DD20934 } \\
\text { (GbPYL6-2D) }\end{array}$ & $\begin{array}{l}\text { AT2G38310 } \\
\text { (AtPYL4)/ } \\
\text { AT5G05440 } \\
\text { (AtPYL5)/ } \\
\text { AT2G40330 } \\
\text { (AtPYL6) }\end{array}$ & & $\begin{array}{l}\text { 29729.m0022 } \\
\text { 90(RcPYRL4) }\end{array}$ & & $\begin{array}{l}\text { BRADI1G16710(BdPYRL5)/ } \\
\text { BRADI1G65130(BdPYRL6) }\end{array}$ & $\begin{array}{l}\text { Os01t0827800 } \\
\text { (OsPYL4)/ } \\
\text { Os05t0473000 } \\
\text { (OsPYL5)/ } \\
\text { Os03t0297600 } \\
\text { (OsPYL6) }\end{array}$ \\
\hline 22 & & & & $\begin{array}{l}\text { AA19258 } \\
\text { (GbPYL9-7A)/ } \\
\text { AA28339 } \\
\text { (GbPYL9-8A) }\end{array}$ & $\begin{array}{l}\text { AT4G01026 } \\
\text { (AtPYL7)/ } \\
\text { AT5G53160 } \\
\text { (AtPYL8)/ } \\
\text { AT1G01360 } \\
\text { (AtPYL9)/ } \\
\text { AT4G27920 } \\
\text { (AtPYL10) }\end{array}$ & $\begin{array}{l}\text { Thecc1EG0 } \\
15359 \\
\text { (TcPYRL8) }\end{array}$ & & $\begin{array}{l}\text { GSVIVT01 } \\
\text { 028704001( } \\
\text { VvPYRL4) }\end{array}$ & & \\
\hline
\end{tabular}


Table 2 (on next page)

Schema of interactions between GhPYLs and GhABIIA/GhABIID. 
Table 2 Schema of interactions between GhPYLs and GhABI1A/GhABI1D

\begin{tabular}{|c|c|c|c|c|c|}
\hline \multirow[b]{2}{*}{ Genes } & \multirow[b]{2}{*}{ Gene ID } & \multicolumn{2}{|l|}{$-\mathbf{A B A}$} & \multicolumn{2}{|l|}{$+\mathbf{A B A}$} \\
\hline & & $\begin{array}{l}\text { GhABI1A } \\
\text { (Gh_A07G01 } \\
\text { 23) }\end{array}$ & $\begin{array}{l}\text { GhABI1D } \\
\text { (Gh_D07G23 } \\
\text { 83) }\end{array}$ & $\begin{array}{l}\text { GhABI1A } \\
\text { (Gh_A07G01 } \\
\text { 23) }\end{array}$ & $\begin{array}{l}\text { GhABI1D } \\
\text { (Gh_D07G23 } \\
\text { 83) }\end{array}$ \\
\hline GhPYR1-1A & Gh_A03G0015 & $\times$ & $x$ & $\sqrt{ }$ & $x$ \\
\hline GhPYR1-1D & Gh_D03G1860 & $\sqrt{ }$ & $\sqrt{ }$ & $\sqrt{ }$ & $\sqrt{ }$ \\
\hline GhPYR1-2A & Gh_A11G0270 & $\times$ & $\times$ & $\times$ & $\times$ \\
\hline GhPYR1-2D & Gh_D11G0290 & $x$ & $\times$ & $\sqrt{ }$ & $\sqrt{ }$ \\
\hline GhPYR1-3A & Gh_A12G1895 & $\sqrt{ }$ & $x$ & $\sqrt{ }$ & $x$ \\
\hline GhPYL2-1A & Gh_A05G0336 & $x$ & $x$ & $\sqrt{ }$ & $\sqrt{ }$ \\
\hline GhPYL2-2A & Gh_A08G2221 & $\times$ & $x$ & $\sqrt{ }$ & $\sqrt{ }$ \\
\hline GhPYL2-2D & Gh_D08G2587 & $\times$ & $\times$ & $\sqrt{ }$ & $\sqrt{ }$ \\
\hline GhPYL2-3D & Gh_D07G0193 & $\times$ & $x$ & $x$ & $x$ \\
\hline GhPYL4-1A & Gh_A01G1990 & $\times$ & $x$ & $\sqrt{ }$ & $\sqrt{ }$ \\
\hline GhPYL4-2A & Gh_A09G2421 & $\sqrt{ }$ & $\sqrt{ }$ & $\sqrt{ }$ & $\sqrt{ }$ \\
\hline GhPYL4-2D & Gh_D01G2250 & $\times$ & $\times$ & $\sqrt{ }$ & $\sqrt{ }$ \\
\hline GhPYL4-3A & Gh_A05G2630 & $x$ & $\times$ & $x$ & $x$ \\
\hline GhPYL6-1D & Gh_D10G2388 & $\sqrt{ }$ & $\times$ & $\sqrt{ }$ & $x$ \\
\hline GhPYL6-2A & Gh_A06G1418 & $\sqrt{ }$ & $\sqrt{ }$ & $\sqrt{ }$ & $\sqrt{ }$ \\
\hline GhPYL6-2D & Gh_D06G1764 & $\sqrt{ }$ & $\sqrt{ }$ & $\sqrt{ }$ & $\sqrt{ }$ \\
\hline GhPYL9-1A & Gh_A08G1117 & $x$ & $\sqrt{ }$ & $\times$ & $\sqrt{ }$ \\
\hline GhPYL9-2A & Gh_A11G0870 & $\sqrt{ }$ & $\times$ & $\sqrt{ }$ & $x$ \\
\hline GhPYL9-3D & Gh_D04G0019 & $\times$ & $x$ & $x$ & $x$ \\
\hline GhPYL9-4D & Gh_D11G0238 & $\sqrt{ }$ & $\sqrt{ }$ & $\sqrt{ }$ & $\sqrt{ }$ \\
\hline GhPYL9-5D & Gh_D12G2306 & $x$ & $\sqrt{ }$ & $\sqrt{ }$ & $\sqrt{ }$ \\
\hline GhPYL9-6A & Gh_A09G1646 & $\sqrt{ }$ & $\sqrt{ }$ & $\sqrt{ }$ & $\sqrt{ }$ \\
\hline GhPYL9-6D & Gh_D09G1740 & $\times$ & $x$ & $x$ & $x$ \\
\hline GhPYL9-7D & Gh_D12G2694 & $\times$ & $x$ & $x$ & $x$ \\
\hline GhPYL11A & Gh_A05G1297 & $\times$ & $\times$ & $\times$ & $\times$ \\
\hline
\end{tabular}

The symbols " $\sqrt{ }$ " and " $x$ " respectively mean "interaction" and "no interaction" between two proteins. "-ABA" and "+ABA" represents experiments performed in the absence or presence of $10 \mu M$ ABA. 